\title{
Search for relativistic magnetic monopoles with the AMANDA-II neutrino telescope
}

\author{
The IceCube Collaboration
}

R. Abbasi ${ }^{1}$, Y. Abdou ${ }^{2}$, T. Abu-Zayyad ${ }^{3}$, J. Adams ${ }^{4}$, J.A. Aguilar ${ }^{1}$, M. Ahlers $^{5}$, K. Andeen ${ }^{1}$, J. Auffenberg $^{6}$, X. Bai $^{7}$, M. Baker ${ }^{1}$, S.W. Barwick ${ }^{8}$, R. Bay ${ }^{9}$, J.L. Bazo Alba ${ }^{10}$, K. Beattie ${ }^{11}$, J.J. Beatty ${ }^{12,13}$, S. Bechet ${ }^{14}$, J.K. Becker ${ }^{15}$, K.-H. Becker ${ }^{6}$, M.L. Benabderrahmane ${ }^{10}$, S. BenZvi ${ }^{1}$, J. Berdermann ${ }^{10}$, P. Berghaus ${ }^{1}$, D. Berley ${ }^{16}$, E. Bernardini ${ }^{10}$, D. Bertrand ${ }^{14}$, D.Z. Besson ${ }^{17}$, M. Bissok ${ }^{18}$, E. Blaufuss ${ }^{16}$, D.J. Boersma ${ }^{18}$, C. Bohm ${ }^{19}$, S. Böser ${ }^{20}$, O. Botner $^{21}$, L. Bradley ${ }^{22}$, J. Braun ${ }^{1}$, S. Buitink ${ }^{11}$, M. Carson ${ }^{2}$, D. Chirkin ${ }^{1}$, B. Christy ${ }^{16}$, J. Clem ${ }^{7}$, F. Clevermann ${ }^{23}$, S. Cohen ${ }^{24}$, C. Colnard ${ }^{25}$, D.F. Cowen ${ }^{22,26}$, M.V. D’Agostino ${ }^{9}$, M. Danninger ${ }^{19}$, J.C. Davis ${ }^{12}$, C. De Clercq ${ }^{27}$, L. Demirörs ${ }^{24}$, O. Depaepe $^{27}$, F. Descamps ${ }^{2}$, P. Desiati ${ }^{1}$, G. de Vries-Uiterweerd ${ }^{2}$, T. DeYoung ${ }^{22}$, J.C. Díaz-Vélez ${ }^{1}$, M. Dierckxsens ${ }^{14}$, J. Dreyer ${ }^{15}$, J.P. Dumm ${ }^{1}$, M.R. Duvoort ${ }^{28}$, R. Ehrlich $^{16}$, J. Eisch ${ }^{1}$, R.W. Ellsworth ${ }^{16}$, O. Engdegård ${ }^{21}$, S. Euler ${ }^{18}$, P.A. Evenson ${ }^{7}$, O. Fadiran ${ }^{29}$, A.R. Fazely ${ }^{30}$, T. Feusels ${ }^{2}$, K. Filimonov ${ }^{9}$, C. Finley ${ }^{19}$, M.M. Foerster ${ }^{22}$, B.D. Fox ${ }^{22}$, A. Franckowiak ${ }^{20}$, R. Franke ${ }^{10}$, T.K. Gaisser ${ }^{7}$, J. Gallagher ${ }^{31}$, M. Geisler $^{18}$, L. Gerhardt ${ }^{11,9}$, L. Gladstone ${ }^{1}$, T. Glüsenkamp ${ }^{18}$, A. Goldschmidt ${ }^{11}$, J.A. Goodman ${ }^{16}$, D. Grant ${ }^{32}$, T. Griesel ${ }^{33}$, A. Groß ${ }^{4,25}$, S. Grullon ${ }^{1}$, M. Gurtner ${ }^{6}$, C. Ha ${ }^{22}$, A. Hallgren ${ }^{21}$, F. Halzen ${ }^{1}$, K. Han ${ }^{4}$, K. Hanson ${ }^{14,1}$, K. Helbing ${ }^{6}$, P. Herquet ${ }^{34}$, S. Hickford ${ }^{4}$, G.C. Hill ${ }^{1}$, K.D. Hoffman ${ }^{16}$, A. Homeier ${ }^{20}$, K. Hoshina ${ }^{1}$, D. Hubert ${ }^{27}$, W. Huelsnitz ${ }^{16}$, J.-P. Hülß ${ }^{18}$, P.O. Hulth ${ }^{19}$, K. Hultqvist ${ }^{19}$, S. Hussain ${ }^{7}$, A. Ishihara ${ }^{35}$, J. Jacobsen ${ }^{1}$, G.S. Japaridze ${ }^{29}$, H. Johansson ${ }^{19}$, J.M. Joseph ${ }^{11}$, K.-H. Kampert ${ }^{6}$, T. Karg ${ }^{6}$, A. Karle ${ }^{1}$, J.L. Kelley ${ }^{1}$, N. Kemming ${ }^{36}$, P. Kenny ${ }^{17}$, J. Kiryluk ${ }^{11,9}$, F. Kislat ${ }^{10}$, S.R. Klein ${ }^{11,9}$, S. Knops ${ }^{18}$, J.-H. Köhne ${ }^{23}$, G. Kohnen ${ }^{34}$, H. Kolanoski ${ }^{36}$, L. Köpke ${ }^{33}$, D.J. Koskinen ${ }^{22}$, M. Kowalski ${ }^{20}$, T. Kowarik ${ }^{33}$, M. Krasberg ${ }^{1}$, T. Krings ${ }^{18}$, G. Kroll ${ }^{33}$, K. Kuehn ${ }^{12}$, T. Kuwabara ${ }^{7}$, M. Labare ${ }^{14}$, S. Lafebre ${ }^{22}$, K. Laihem ${ }^{18}$, H. Landsman ${ }^{1}$, R. Lauer ${ }^{10}$, R. Lehmann ${ }^{36}$, D. Lennarz ${ }^{18}$, J. Lünemann ${ }^{33}$, J. Madsen ${ }^{3}$, P. Majumdar ${ }^{10}$, A. Marotta $^{14}$, R. Maruyama ${ }^{1}$, K. Mase ${ }^{35}$, H.S. Matis ${ }^{11}$, M. Matusik ${ }^{6}$, K. Meagher ${ }^{16}$, M. Merck $^{1}$, P. Mészáros ${ }^{26,22}$, T. Meures $^{18}$, E. Middell ${ }^{10}$, N. Milke ${ }^{23}$, J. Miller ${ }^{21}$, T. Montaruli ${ }^{1,37}$, R. Morse ${ }^{1}$, S.M. Movit ${ }^{26}$, R. Nahnhauer ${ }^{10}$, J.W. Nam ${ }^{8}$, U. Naumann ${ }^{6}$, P. Nießen ${ }^{7}$, D.R. Nygren ${ }^{11}$, S. Odrowski ${ }^{25}$, A. Olivas ${ }^{16}$, M. Olivo ${ }^{21,15}$, A. O’Murchadha ${ }^{1}$, M. Ono ${ }^{35}$, S. Panknin ${ }^{20}$, L. Paul ${ }^{18}$, C. Pérez de los Heros ${ }^{21}$, J. Petrovic ${ }^{14}$, A. Piegsa ${ }^{33}$, D. Pieloth ${ }^{23}$, R. Porrata ${ }^{9}$, J. Posselt ${ }^{6}$, P.B. Price ${ }^{9}$, M. Prikockis ${ }^{22}$, G.T. Przybylski ${ }^{11}$, K. Rawlins ${ }^{38}$,

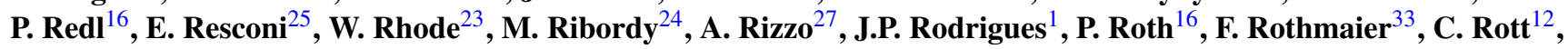
C. Roucelle $^{25}$, T. Ruhe ${ }^{23}$, D. Rutledge ${ }^{22}$, B. Ruzybayev ${ }^{7}$, D. Ryckbosch ${ }^{2}$, H.-G. Sander ${ }^{33}$, M. Santander ${ }^{1}$, S. Sarkar ${ }^{5}$, K. Schatto ${ }^{33}$, S. Schlenstedt ${ }^{10}$, T. Schmidt ${ }^{16}$, A. Schukraft ${ }^{18}$, A. Schultes ${ }^{6}$, O. Schulz ${ }^{25}$, M. Schunck ${ }^{18}$, D. Seckel ${ }^{7}$, B. Semburg ${ }^{6}$, S.H. Seo ${ }^{19}$, Y. Sestayo ${ }^{25}$, S. Seunarine ${ }^{39}$, A. Silvestri ${ }^{8}$, A. Slipak ${ }^{22}$, G.M. Spiczak ${ }^{3}$, C. Spiering $^{10}$, M. Stamatikos ${ }^{12,40}$, T. Stanev ${ }^{7}$, G. Stephens ${ }^{22}$, T. Stezelberger ${ }^{11}$, R.G. Stokstad ${ }^{11}$, S. Stoyanov ${ }^{7}$, E.A. Strahler ${ }^{27}$, T. Straszheim ${ }^{16}$, G.W. Sullivan ${ }^{16}$, Q. Swillens ${ }^{14}$, H. Taavola ${ }^{21}$, I. Taboada ${ }^{41}$, A. Tamburro ${ }^{3}$, O. Tarasova ${ }^{10}$, A. Tepe $^{41}$, S. Ter-Antonyan ${ }^{30}$, S. Tilav ${ }^{7}$, P.A. Toale ${ }^{22}$, S. Toscano ${ }^{1}$, D. Tosi ${ }^{10}$, D. Turčan ${ }^{16}$, N. van Eijndhoven ${ }^{27}$,

J. Vandenbroucke ${ }^{9}$, A. Van Overloop ${ }^{2}$, J. van Santen ${ }^{1}$, M. Voge $^{25}$, B. $_{\text {Voigt }}{ }^{10}$, C. Walck $^{19}$, T. Waldenmaier $^{36}$, M. Wallraff ${ }^{18}$, M. Walter ${ }^{10}$, Ch. Weaver ${ }^{1}$, C. Wendt $^{1}$, S. Westerhoff ${ }^{1}$, N. Whitehorn ${ }^{1}$, K. Wiebe ${ }^{33}$, C.H. Wiebusch $^{18}$, G. Wikström ${ }^{19}$, D.R. Williams ${ }^{42}$, R. Wischnewski ${ }^{10}$, H. Wissing ${ }^{16, a}$, M. Wolf ${ }^{25}$, K. Woschnagg ${ }^{9}$ C. Xu ${ }^{7}, X$.W. Xu ${ }^{30}$, G. Yodh $^{8}$, S. Yoshida ${ }^{35}$, P. Zarzhitsky ${ }^{42}$

\footnotetext{
${ }^{1}$ Dept. of Physics, University of Wisconsin, Madison, WI 53706, USA

${ }^{2}$ Dept. of Subatomic and Radiation Physics, University of Gent, 9000 Gent, Belgium

${ }^{3}$ Dept. of Physics, University of Wisconsin, River Falls, WI 54022, USA

${ }^{4}$ Dept. of Physics and Astronomy, University of Canterbury, Private Bag 4800, Christchurch, New Zealand

${ }^{5}$ Dept. of Physics, University of Oxford, 1 Keble Road, Oxford OX1 3NP, UK

${ }^{6}$ Dept. of Physics, University of Wuppertal, 42119 Wuppertal, Germany

${ }^{7}$ Bartol Research Institute and Department of Physics and Astronomy, University of Delaware, Newark, DE 19716, USA

${ }^{8}$ Dept. of Physics and Astronomy, University of California, Irvine, CA 92697, USA

${ }^{9}$ Dept. of Physics, University of California, Berkeley, CA 94720, USA

${ }^{10}$ DESY, 15735 Zeuthen, Germany

${ }^{11}$ Lawrence Berkeley National Laboratory, Berkeley, CA 94720, USA
} 
${ }^{12}$ Dept. of Physics and Center for Cosmology and Astro-Particle Physics, Ohio State University, Columbus, OH 43210, USA

${ }^{13}$ Dept. of Astronomy, Ohio State University, Columbus, OH 43210, USA

${ }^{14}$ Science Faculty CP230, Université Libre de Bruxelles, 1050 Brussels, Belgium

${ }^{15}$ Fakultät für Physik \& Astronomie, Ruhr-Universität Bochum, 44780 Bochum, Germany

${ }^{16}$ Dept. of Physics, University of Maryland, College Park, MD 20742, USA

${ }^{17}$ Dept. of Physics and Astronomy, University of Kansas, Lawrence, KS 66045, USA

${ }^{18}$ III. Physikalisches Institut, RWTH Aachen University, 52056 Aachen, Germany

${ }^{19}$ Oskar Klein Centre and Dept. of Physics, Stockholm University, SE 10691 Stockholm, Sweden

${ }^{20}$ Physikalisches Institut, Universität Bonn, Nussallee 12, 53115 Bonn, Germany

${ }^{21}$ Dept. of Physics and Astronomy, Uppsala University, Box 516, 75120 Uppsala, Sweden

${ }^{22}$ Dept. of Physics, Pennsylvania State University, University Park, PA 16802, USA

${ }^{23}$ Dept. of Physics, TU Dortmund University, 44221 Dortmund, Germany

${ }^{24}$ Laboratory for High Energy Physics, École Polytechnique Fédérale, 1015 Lausanne, Switzerland

${ }^{25}$ Max-Planck-Institut für Kernphysik, 69177 Heidelberg, Germany

${ }^{26}$ Dept. of Astronomy and Astrophysics, Pennsylvania State University, University Park, PA 16802, USA

${ }^{27}$ Dienst ELEM, Vrije Universiteit Brussel, 1050 Brussels, Belgium

${ }^{28}$ Dept. of Physics and Astronomy, Utrecht University/SRON, 3584 CC Utrecht, The Netherlands

${ }^{29}$ CTSPS, Clark-Atlanta University, Atlanta, GA 30314, USA

${ }^{30}$ Dept. of Physics, Southern University, Baton Rouge, LA 70813, USA

${ }^{31}$ Dept. of Astronomy, University of Wisconsin, Madison, WI 53706, USA

${ }^{32}$ Dept. of Physics, University of Alberta, Edmonton, Alberta, Canada T6G 2G7

${ }^{33}$ Institute of Physics, University of Mainz, Staudinger Weg 7, 55099 Mainz, Germany

${ }^{34}$ Université de Mons, 7000 Mons, Belgium

${ }^{35}$ Dept. of Physics, Chiba University, Chiba 263-8522, Japan

${ }^{36}$ Institut für Physik, Humboldt-Universität zu Berlin, 12489 Berlin, Germany

${ }^{37}$ Dipartimento di Fisica, Università di Bari and Sezione INFN 70126, Bari, Italy

${ }^{38}$ Dept. of Physics and Astronomy, University of Alaska Anchorage, 3211 Providence Dr., Anchorage, AK 99508, USA

${ }^{39}$ Dept. of Physics, University of the West Indies, Cave Hill Campus, Bridgetown 11000, Barbados

${ }^{40}$ NASA Goddard Space Flight Center, Greenbelt, MD 20771, USA

${ }^{41}$ School of Physics and Center for Relativistic Astrophysics, Georgia Institute of Technology, Atlanta, GA 30332, USA

${ }^{42}$ Dept. of Physics and Astronomy, University of Alabama, Tuscaloosa, AL 35487, USA

Received: 23 June 2010 / Revised: 22 July 2010 / Published online: 28 August 2010

(C) The Author(s) 2010. This article is published with open access at Springerlink.com

Abstract We present the search for Cherenkov signatures from relativistic magnetic monopoles in data taken with the AMANDA-II detector, a neutrino telescope deployed in the Antarctic ice cap at the Geographic South Pole. The nonobservation of a monopole signal in data collected during the year 2000 improves present experimental limits on the flux of relativistic magnetic monopoles: Our flux limit varies between $3.8 \times 10^{-17} \mathrm{~cm}^{-2} \mathrm{~s}^{-1} \mathrm{sr}^{-1}$ (for monopoles moving at the vacuum speed of light) and $8.8 \times 10^{-16} \mathrm{~cm}^{-2} \mathrm{~s}^{-1} \mathrm{sr}^{-1}$ (for monopoles moving at a speed $\beta=v / c=0.76$, just above the Cherenkov threshold in ice). These limits apply to monopoles that are energetic enough to penetrate the Earth and enter the detector from below the horizon. The limit obtained for monopoles reaching the detector from above the horizon is less stringent by roughly an order of magnitude, due to the much larger background from down-going atmospheric muons. This looser limit is however valid for a larger class of magnetic monopoles, since the monopoles are not required to pass through the Earth.

a e-mail: hwissing@icecube.umd.edu

\section{Introduction}

The existence of magnetic monopoles, particles carrying magnetic charge, was hypothesized in various theoretical contexts. When added to classical electrodynamics, magnetic charges would symmetrize Maxwell's Equations with respect to the sources of the electromagnetic field. In a quantum-mechanical context, their existence requires the quantization of both electric and magnetic charge [1]. The magnetic elementary charge, the so-called Dirac Charge $g_{\mathrm{D}}$, takes the value

$g_{\mathrm{D}}=\frac{e}{2 \alpha}$,

where $e$ is the electric elementary charge and $\alpha$ is the finestructure constant. In the context of Grand Unified Theories (GUTs) [2], magnetic monopoles appear as stable, finite energy solutions of the field equations [3, 4].

If hot big bang cosmology is correct, GUT monopoles would have been created in the early universe during phase transitions associated with the spontaneous breakdown of the unified gauge symmetry [5]. The mass and the charge of monopoles in a particular GUT depend on the underlying gauge group, its symmetry breaking hierarchy, and on 
the type and the temperature of the phase transition. Predicted masses range from $10^{5}$ to $10^{17} \mathrm{GeV}$ [6-10]; monopole charges are integer multiples of the Dirac charge.

Magnetic monopoles are stable and those monopoles created in the early universe should still be present in cosmic radiation. The monopole number density depends on whether or not the universe underwent an inflationary epoch and on whether the monopoles were created before, during or after this epoch [11].

Due to the large numerical value of the magnetic charge, monopoles are efficiently accelerated in large scale cosmic magnetic fields. The kinetic energy acquired on passing through a magnetic field $\boldsymbol{B}$ is $E_{\mathrm{kin}}=g \int_{\text {path }} \boldsymbol{B} \cdot d \boldsymbol{l}$, with $g$ being the magnetic charge, an integer multiple of $g_{\mathrm{D}}$. During their lifetime, magnetic monopoles should have acquired kinetic energies of the order of $10^{15} \mathrm{GeV}$, on average [10]. Consequently, monopoles with masses at or below this energy scale should have been accelerated to relativistic velocities [10]. The acceleration of magnetic monopoles in the Galactic magnetic field also implies a generic upper bound on monopole flux: the requirement that the Galactic magnetic field is not depleted faster than it can be regenerated constrains the monopole flux to be less than $10^{-15} \mathrm{~cm}^{-2} \mathrm{~s}^{-1} \mathrm{sr}^{-1}$. This is the oft-quoted Parker Bound $[12,13]$.

Over the last three decades, relic monopoles have been searched for by a number of experiments, but so far, there is no experimental proof of their existence. Today, the flux of magnetic monopoles is experimentally constrained to a level $\mathcal{O}\left(10^{-16} \mathrm{~cm}^{-2} \mathrm{~s}^{-1} \mathrm{sr}^{-1}\right)$ [13-15], so only extremely large detectors have the potential to detect a magnetic monopole.

Large Cherenkov telescopes built in open, naturally occurring media like sea water or glacial ice were originally constructed to detect Cherenkov light from electrically charged secondary particles produced in rare interactions of high energy extraterrestrial neutrinos with the surrounding matter. These telescopes could also detect magnetic monopoles with specific properties: (1) Relativistic monopoles moving at a speed above the Cherenkov threshold of the utilized medium could efficiently be detected via their direct Cherenkov emissions [17], or (2) monopoles that catalyze the decay of nucleons in the target matter-a property that is predicted for a large class of GUT monopoles [18, 19]—could be detected via the Cherenkov emissions from secondary particles produced in consecutive nucleon decays along the monopole trajectory. For Cherenkov telescopes, the two detection channels rely on very different monopole signatures and are investigated in complementary efforts.

This paper describes a search for relativistic magnetic monopoles with the Antarctic Muon And Neutrino Detection Array (AMANDA-II), an under-ice neutrino telescope that was operated during the years 2000 to 2009 at the South Pole [23]. ${ }^{1}$

The analysis was developed using data taken during AMANDA-II's first year of operation. Although several more years of data are available, and the analysis could be applied also to data taken during later years, only data taken during the year 2000 has been searched for Cherenkov signals from magnetic monopoles. The remaining years will probably remain unanalyzed. The filtering of multiple years of data and the necessary Monte Carlo simulations, which take into account small changes in the detector set up over the years, would not be complete before the presently under construction IceCube detector, the successor to AMANDAII with much better sensitivity, will deliver first results on magnetic monopoles.

Nevertheless, the monopole search with one year of AMANDA-II data presented here, still improves current experimental bounds on the flux of magnetic monopoles.

\section{Monopole energy loss in matter}

Relativistic magnetic monopoles passing through matter lose energy via electromagnetic, strong, and weak interactions [10]. The electromagnetic interaction of monopoles is, except for the much larger numerical value of the coupling constant, similar to the interaction of electrically charged particles. Therefore the electromagnetic energy loss is fairly well understood.

Like electrically charged particles, monopoles lose energy through ionization and excitations of atoms in the target material ("collisional" energy loss), and through stochastic energy loss processes $\left(e^{+} e^{-}\right.$pair production, bremsstrahlung, and photo-nuclear interactions). The collisional energy loss depends only weakly on the monopole's Lorentz boost and dominates stochastic energy losses up to Lorentz boosts of $\Gamma<10^{3}[10,16]$. For higher Lorentz boosts, the stochastic energy losses become dominant and cause the total energy loss to rise steeply with $\Gamma$. Figure 1 shows the total average energy loss of relativistic magnetic monopoles in various media, according to approximate energy loss formulas given in [16] (collisional energy loss) and [10] (stochastic energy loss).

Contrary to the electromagnetic energy loss, strong and weak energy loss processes are not well understood. While the weak interaction loss is believed to be negligible small, the strong interaction energy losses may be significant [10]. However, due to the lack of a definite theoretical description, the strong interaction energy loss is often ignored in the literature, and will be ignored in the following discussion as

\footnotetext{
${ }^{1}$ The search for nucleon-decay-catalyzing monopoles with AMANDAII is described in reference [20,21].
} 


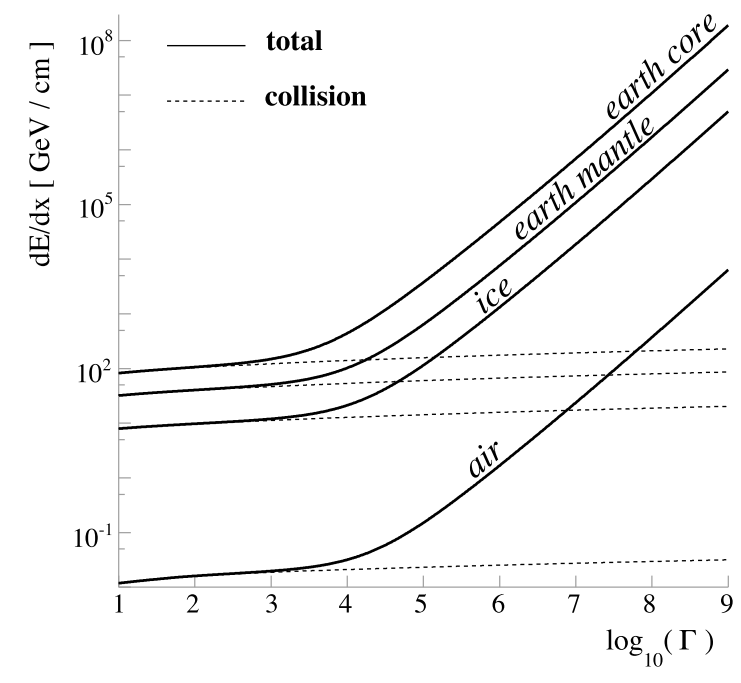

Fig. 1 Average electromagnetic energy loss of relativistic magnetic monopoles in the Earth core, the Earth mantle, ice, and air as a function of the Lorentz boost $\Gamma$. The full lines are the total energy loss, i.e., the sum of collisional loss, $e^{+} e^{-}$pair production, bremsstrahlung, and photo-nuclear interactions according to reference [10]. The dashed lines are the collisional energy loss only [16]

well. We bear in mind, however, that the strong interaction energy loss may be as large as the electromagnetic one [10].

\subsection{Cherenkov radiation}

A small fraction of the electromagnetic energy loss is due to Cherenkov radiation. The Cherenkov emission of magnetically charged particles is much more intense than that of electrically charged particles and enable efficient detection of relativistic magnetic monopoles with a neutrino telescope.

The number of Cherenkov photons $N_{\gamma}$ per path length $d x$ and photon wavelength $d \lambda$ emitted by a magnetic charge $g$ passing through matter with index of refraction $n$ is [17]

$\frac{d N_{\gamma}}{d x d \lambda}=\frac{2 \pi \alpha}{\lambda^{2}}\left(\frac{g n}{e}\right)^{2}\left(1-\frac{1}{\beta^{2} n^{2}}\right)$

where $\beta$ is the speed of the monopole as a fraction of the speed of light in vacuum.

Passing through ice $(n \approx 1.33)$, a minimally charged relativistic monopole will emit $\mathcal{O}\left(10^{6}\right)$ Cherenkov photons per centimeter in the wavelength interval between $400 \mathrm{~nm}$ and $600 \mathrm{~nm}$, where the AMANDA-II detector is sensitive. This is a factor $\left(g_{D} \cdot n / e\right)^{2}=(n / 2 \alpha)^{2} \approx 8300$ larger than the number of Cherenkov photons emitted from a bare relativistic muon. Cherenkov emissions of highly relativistic monopoles, for which stochastic processes dominate the energy loss, are enhanced by Cherenkov light from relativistic electrically charged secondary particles that are produced along the monopole track.
2.2 Monopole passage through the Earth

For the search for relativistic monopoles with AMANDA-II, the total energy loss is decisive for the signal acceptance. Full acceptance $(4 \pi \mathrm{sr})$ is reached if the monopoles have sufficient kinetic energy to traverse the full diameter of the Earth and enter the detector vertically from below at a relativistic speed. Half the acceptance $(2 \pi \mathrm{sr})$ is reached if monopoles are still sufficiently relativistic after penetrating $\sim 150 \mathrm{~km}$ of ice, the matter overburden that must be crossed to horizontally reach the center of the detector at a depth of $1730 \mathrm{~m}$.

The Cherenkov threshold in ice is $\beta>1 / n=0.75$, corresponding to a minimum Lorentz boost $\Gamma>1.51$. In order to reach the detector with at least this boost factor, a monopole with mass $M$ must arrive at the Earth with a Lorentz boost of

$\Gamma_{\text {initial }}>\frac{\Delta E_{\text {total }}}{M}+1.51$,

where $\Delta E_{\text {total }}$ is the energy lost during the passage through the matter overburden above or below the detector. Since $\Gamma=E / M$, the above relation in combination with the energy loss function dictates the minimum mass and the minimum initial kinetic energy a monopole must have to be detectable with AMANDA-II.

Both the monopole mass and the initial kinetic energy are unknown. Although the monopole mass can be predicted in a specific GUT, the mass predictions from various proposed GUTs span many orders of magnitudes. Even if we were to test one specific GUT for which the monopole mass is fixed, the expected monopole kinetic energy remains highly speculative as it depends on the abundance, strengths, and spatial extensions of cosmic magnetic fields. In magnetic fields of ordinary galaxies, monopoles would gain an energy of roughly $10^{11} \mathrm{GeV}$ [24]. However, during their lifetimes, monopoles should have acquired much higher kinetic energies in larger scale extragalactic structures. Monopoles today are expected to have a broad energy distribution with an estimated average around $10^{15} \mathrm{GeV}$ [10].

In order to determine the mass range and the range of initial kinetic energies for which monopoles are capable of penetrating through the Earth and reach AMANDA-II at a sufficiently relativistic speed, we have integrated the energy loss formulas for the average electromagnetic energy loss given in [10] over the approximate matter density profile of the Earth (Fig. 2).

The angular regions over which monopoles with masses between $10^{5}$ and $10^{18} \mathrm{GeV}$ and initial kinetic energies between $10^{5}$ and $10^{18} \mathrm{GeV}$ can reach the center of AMANDAII at a speed above the Cherenkov threshold $(\beta>0.75)$ are shown in Fig. 3. For kinetic energies of $10^{15} \mathrm{GeV}$ or greater, the full signal acceptance of $4 \pi \mathrm{sr}$ is reached for monopoles 


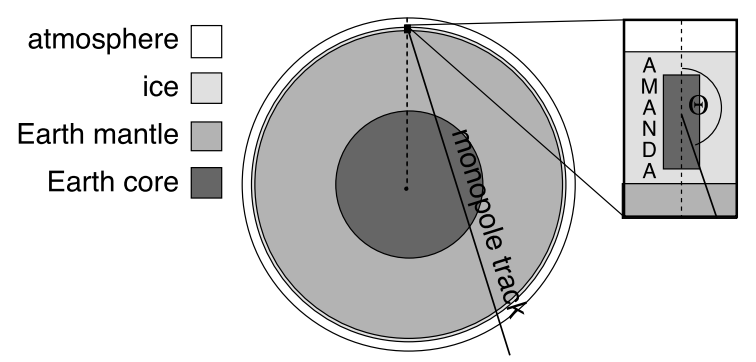

Fig. 2 Schematic sketch (not to scale) of the approximate matter profile of the Earth that was used to determine the total energy loss of monopoles passing through the Earth. The Earth interior is approximated by two shells, a core with radius $R_{\text {core }}=3.486 \times 10^{6} \mathrm{~m}$ and density $\rho_{\text {core }}=11.5 \mathrm{~g} / \mathrm{cm}^{3}$, and a mantle which extends to $R_{\text {mantle }}=6.371 \times 10^{6} \mathrm{~m}$ and $\rho_{\text {mantle }}=4.0 \mathrm{~g} / \mathrm{cm}^{3}[10]$. The outer layers, which are only relevant for monopole tracks with zenith angles $\Theta<90^{\circ}$, are $2800 \mathrm{~m}$ ice $\left(\rho_{\text {ice }}=0.9 \mathrm{~g} / \mathrm{cm}^{3}\right.$ [22] $)$ and $20 \mathrm{~km}$ air $\left(\rho_{\text {air }}=1.2 \times 10^{-3} \mathrm{~g} / \mathrm{cm}^{3}[22]\right)$

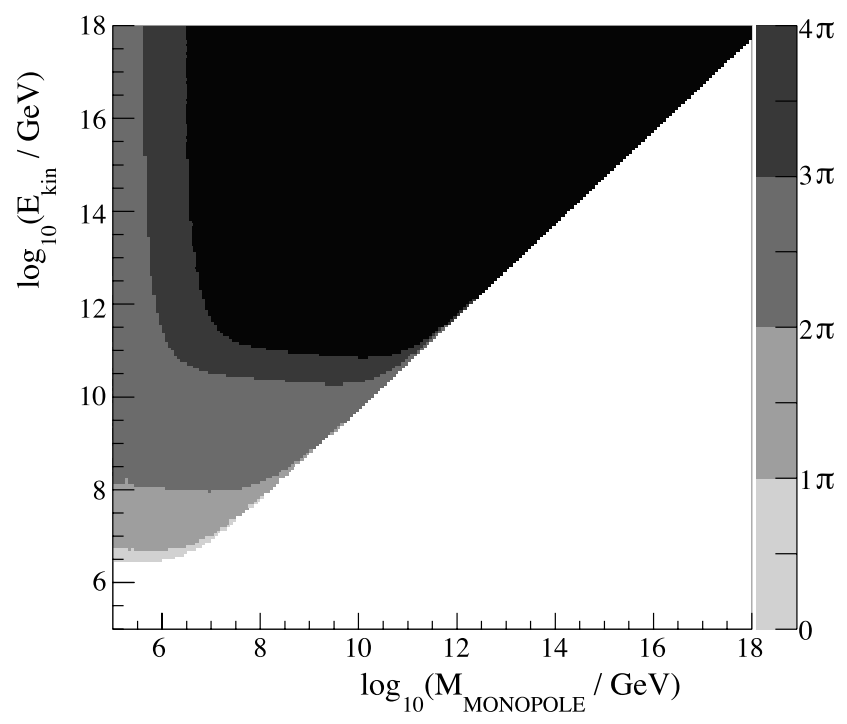

Fig. 3 Angular acceptance of AMANDA-II to relativistic magnetic monopoles. The grey shaded areas represent the regions of monopole mass and kinetic energy for which monopoles are capable of reaching the detector with a speed above the Cherenkov threshold $(\beta=0.75)$. The darkest area corresponds to the region of full acceptance, i.e., to the region where monopoles have a speed $\beta>0.75$ after having traversed the full diameter of the Earth and can hence be detected over a solid angle of $\Omega=4 \pi$ sr. The lighter areas correspond to the regions of $4 \pi \mathrm{sr}>\Omega \geq 3 \pi \mathrm{sr}, 3 \pi \mathrm{sr}>\Omega \geq 2 \pi \mathrm{sr}, 2 \pi \mathrm{sr}>\Omega \geq \pi \mathrm{sr}$, and $\pi \mathrm{sr}>\Omega>0$ angular acceptance

with masses greater than $\sim 10^{7} \mathrm{GeV}$. Lighter monopoles can still be detected with a limited angular acceptance.

\section{The AMANDA-II neutrino telescope}

AMANDA-II was an under-ice neutrino telescope at the geographic South Pole. The 2800 meter-thick ice sheet [25] covering the Antarctic continent at the South Pole served
AMANDA-II as Cherenkov medium and target material to detect rare interactions of highly energetic neutrinos [26]. Cherenkov photons from electrically charged secondary particles were observed using a three dimensional grid of light sensitive optical modules deployed at depths below $1500 \mathrm{~m}$.

Although the surface electronics and the data acquisition system was decommissioned in late 2009, the under-ice hardware remained frozen in place. AMANDA-II comprises a total of 677 light sensitive optical modules (OMs) that are located within an approximately cylindrical volume of $200 \mathrm{~m}$ diameter and $500 \mathrm{~m}$ height. The OMs were deployed in 19 vertical strings, arranged in three concentric circles (Fig. 4).

Each OM consists of a photo-multiplier tube (PMT) and supporting electronics enclosed in a transparent pressure sphere. Power was supplied from the surface via electrical cables, which in some strings were also used for the PMT signal transmission. Other strings were read out via optical fiber.

Starting from the detector center, the 19 strings were deployed during the Austral summers 1995/1996 to $1999 / 2000$. In between these deployment campaigns, the PMT signal transmission techniques were further developed so that the later deployed strings, which define the surface of the cylindrical detector, had improved PMT signal transmission using optical fiber. The fibers are essentially dispersionfree, which resulted in a better resolution of multiple subsequent PMT pulses [27].

The low dark noise rate of the OMs that operated in a cold and sterile environment permitted the use of a simple majority trigger, which was implemented in the surface electronics. The trigger condition used for this analysis required a minimum of $24 \mathrm{OMs}$ hit in a fixed coincidence window of $2.5 \mu \mathrm{s}$. For each triggered event, leading and trailing edge times of up to eight PMT pulses and one peak amplitude were recorded per OM.

The timing and spatial pattern of the PMT pulses recorded during a particle crossing allows reconstruction of the particle direction. The total number and the amplitudes of the PMT pulses provide a measure of the amount of Cherenkov light that was deposited in the detector.

\section{The atmospheric muon background}

The vast majority of events recorded by AMANDA-II were triggered by muons created in interactions of high energy cosmic rays in the atmosphere above the detector. The energy spectrum of atmospheric muons at the surface is steeper than the spectrum of the primary cosmic rays by approximately one power in energy [28]. Only those muons which reach the surface with energies of at least a few hundreds of $\mathrm{GeV}$ can penetrate to AMANDA-II depths and could cause 
Fig. 4 Arrangement of the 19 AMANDA-II strings in the horizontal plane. The strings are arranged in three concentric circles, with one string in the center. The strings on the inner two circles (strings 1-10) are read out via electrical cables, the strings on the outermost circle (strings 11-19) are read out via optical fiber electrical readout (coaxial cable) deployed 1995/96

electrical readout (twisted pair cable) deployed 1996/97

optical readout deployed 1997-2000

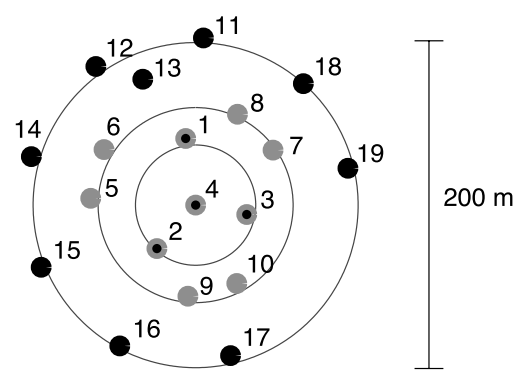

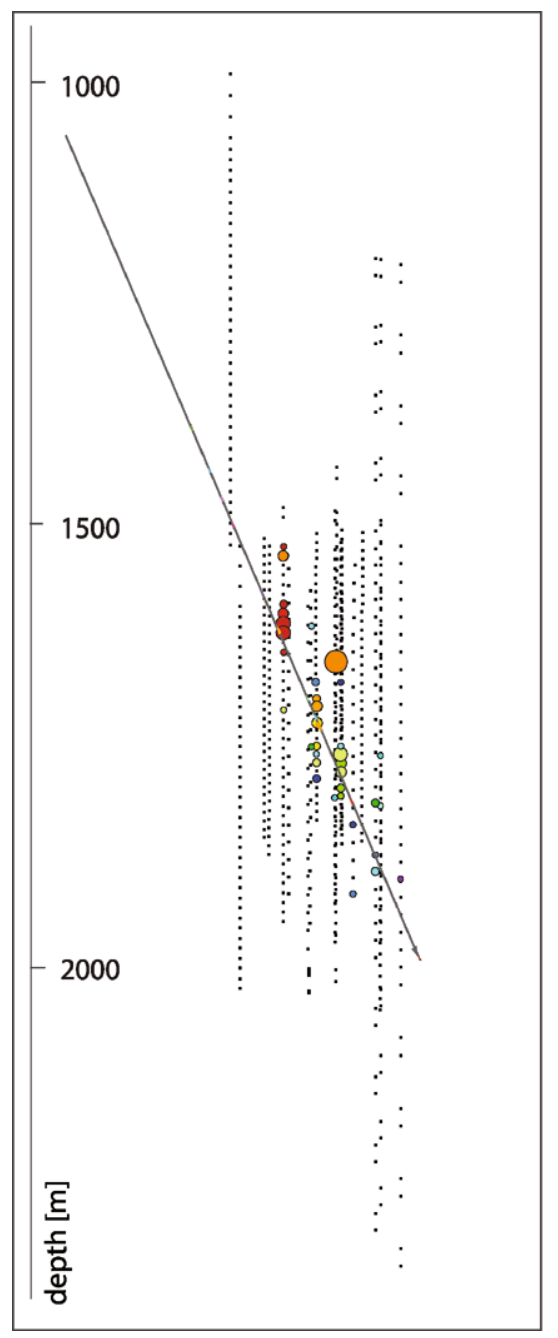

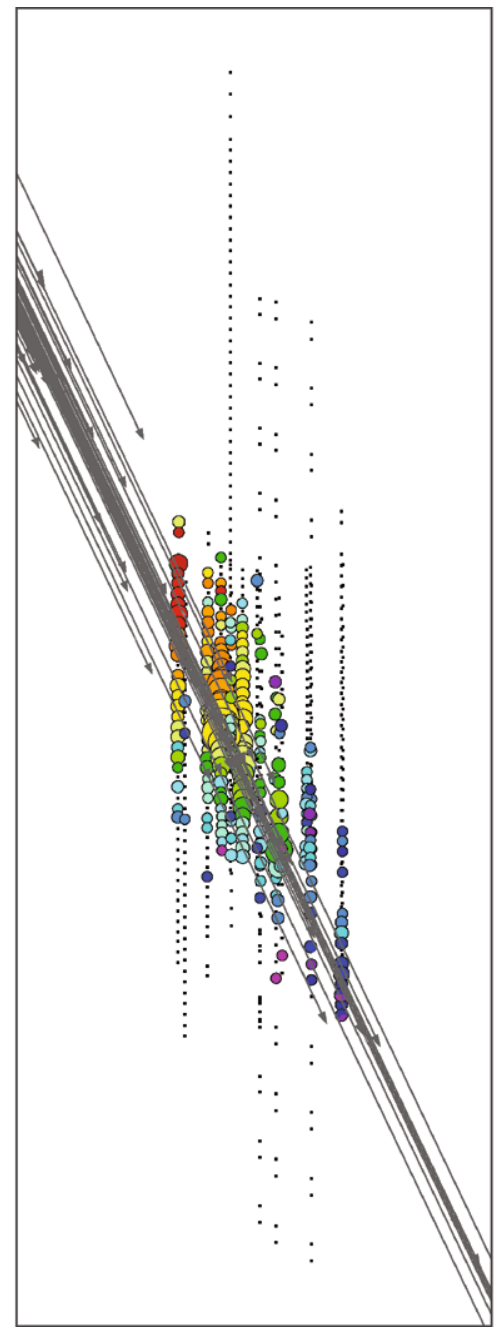

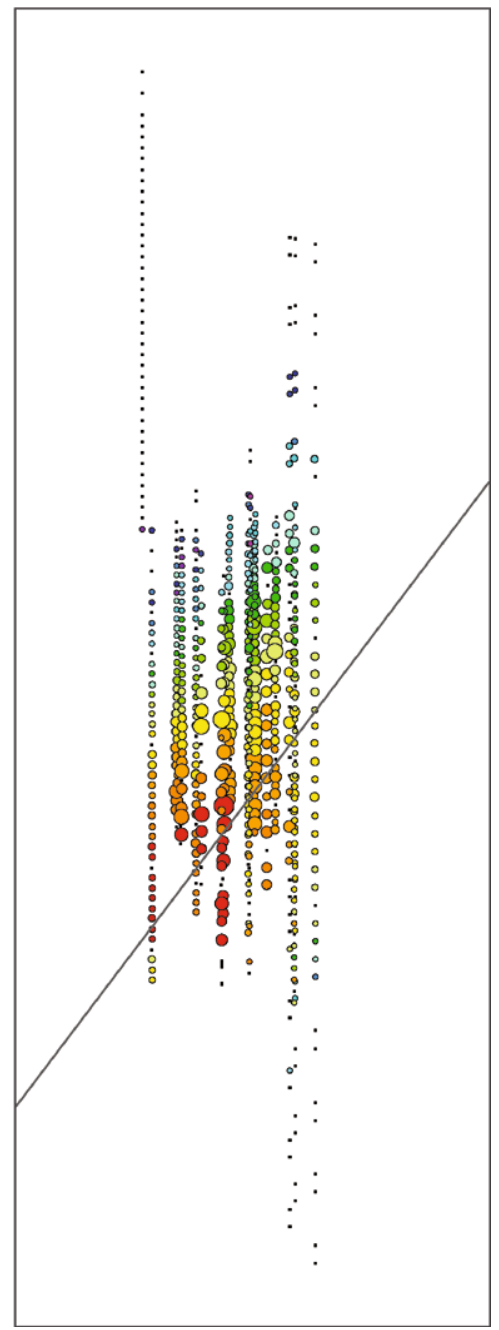

Fig. 5 Simulated Cherenkov signatures of background atmospheric muons and a relativistic monopole in the AMANDA-II event display. The locations of the OMs are marked as black dots. The particle tracks are drawn as grey lines or arrows (the arrow heads indicate the points where muons have ranged out). The colored circles mark OMs that have registered photons. The arrival times of the first photon in each

a trigger. The 24-fold majority trigger described in the previous section resulted in an average event rate of roughly $80 \mathrm{~Hz}$ due to atmospheric muons.

The Cherenkov light yield of atmospheric muons and secondary particles created along the muon track rises with
$\mathrm{OM}$ are represented as colors from red (early hit) to violet (late hit), and the sizes of the circles are a measure for the peak amplitudes of the PMT pulses. Left: Single atmospheric muon induced by a $34 \mathrm{TeV}$ cosmic ray proton. Middle: Muon bundle induced by a $40 \mathrm{PeV}$ iron nucleus. Right: Up-going relativistic magnetic monopole with speed $\beta \approx 1$

the muon energy. For muon energies above $10 \mathrm{PeV}$, the total light yield becomes comparable to that of a relativistic magnetic monopole. The probability that individual muons to reach AMANDA-II with PeV energies is extremely small. However, high energy cosmic ray collisions in the at- 
mosphere produce "bundles" of many nearly parallel muons that simultaneously reach AMANDA-II and spread Cherenkov light over large areas in the detector. Such muon bundles are the dominant background to monopole searches with AMANDA-II. Figure 5 shows the Cherenkov signatures of a single simulated atmospheric muon, a large simulated atmospheric muon bundle, and a simulated relativistic magnetic monopole in the AMANDA-II detector.

\section{Event selection strategy}

Apart from the Cherenkov light intensity, magnetic monopoles and atmospheric muons differ in their arrival directions. While atmospheric muons can enter the detector only from above the horizon, magnetic monopoles with sufficient mass and kinetic energy can arrive from all directions. The only Standard Model particles that could enter AMANDAII from below the horizon and leave an "up-going" Cherenkov light pattern are muons or tau leptons produced by highly energetic neutrinos after having crossed the Earth. Up-going neutrino induced muons, however, will have insufficient Cherenkov light yield to mimic a relativistic magnetic monopole, as neutrinos with $\mathrm{PeV}$ energies will be absorbed in the Earth. Therefore, the search for monopoles below the horizon (i.e., the search for monopoles coming from the northern hemisphere) has very little background, the only significant background being those down-going muons bundles which are mis-reconstructed as up-going. This background can be eliminated with relatively soft criteria related to the reconstruction accuracy and the event light yield.

For the search for magnetic monopoles above the horizon (i.e., the for monopoles coming from the southern hemisphere), the light yield is the main distinctive feature to separate signal from background. Directional information is however still beneficial, because the correlation between Cherenkov light yield and particle direction differs for magnetic monopoles and muon bundles. While heavy magnetic monopoles lose only a small fraction of their kinetic energies on traversing the ice overburden above the detector, muons will lose a substantial fraction of their initial energy, and a fraction of the muons contained in a bundle will range out before reaching the detector. As the energy lost upon reaching the detector rises with the amount of matter traversed, the light yield from muon bundles strongly depends on the zenith angle. Event selection criteria that combine the directional information from a track reconstruction with the light yield enable a reduction of the atmospheric muon background to a few events per year.

Because the searches for magnetic monopoles coming from the northern and southern hemispheres have very different background expectations, event selection criteria are optimized separately for both angular regions.

\section{Experimental and simulated data sets}

This analysis is developed using simulated data while the experimental data are kept blind. In order to ensure that the detector simulation provides an accurate description of the detector response, $20 \%$ of the experimental data are used for comparisons between experimental and simulated data throughout the development of the analysis. This $20 \%$ subset (composed of data taken on roughly every fifth day of the year) is later discarded and the developed selection criteria are applied to the complementary $80 \%$ of the data.

\subsection{The experimental data set}

This analysis concerns the data collected between February and November 2000. The total data taking time amounts to 248.3 days. After subtraction of detector dead time ( $~ 17 \%)$ and exclusion of data of insufficient quality [29], 193 days of effective livetime remain. A total of 155 days of livetime ( $80 \%$ of this data) are used in the final analysis.

\subsection{Simulation of atmospheric muons}

Atmospheric muons are generated with corsika [30], a program to simulate air showers induced by cosmic ray nuclei with energies up to $10^{11} \mathrm{GeV}$ and masses up to $A=56$ (iron). For this work, air showers are simulated using the QGSJET01 [31] hadronic interaction model.

In order to reproduce the atmospheric muon flux over the relevant energy range, we use two different parameterizations of the energy spectrum and mass composition of the cosmic-ray spectrum:

(1) The poly-gonato model [32]. This model assumes the flux of galactic cosmic rays to contain all elements of the periodic table up to uranium. According to the model, elements heavier than iron contribute significantly $(>10 \%)$ to the total flux at primary energies above $\sim 50 \mathrm{PeV}$, and dominate the spectrum at energies above $\sim 200 \mathrm{PeV}$. Therefore, simulations of the all-particle cosmic ray flux utilizing corsika (which does not support the simulation of the trans-iron elements) are accurate only in the energy range below $\sim 50 \mathrm{PeV}$. We use simulations of the poly-gonato model to predict the background at those analysis levels at which more than $99 \%$ of the expected atmospheric muon background is induced by cosmic-ray primaries with energies below $50 \mathrm{PeV}$. This is the case for all analysis levels, except for the last analysis levels for the monopole search above the horizon.

(2) A two-component model [33], which approximates the all particle cosmic ray flux as being composed of only two elements, protons and iron, with both components following a broken power-law energy spectrum. ${ }^{2}$ Simulations

\footnotetext{
${ }^{2}$ The parameters of the power-law spectra are fitted to air-shower data taken with the KASCADE [34] detector.
} 
of the two-component-model provide a good description of atmospheric muon background in the $\mathrm{PeV}$ to $\mathrm{EeV}$ energy range and are used for the monopole search above the horizon from the penultimate analysis level on.

The generation of the atmospheric muon background uses importance sampling in energy so that at the final analysis level the statistical error in the background prediction is of the order of the systematic error or less.

The muon component of the generated air-showers is passed to the muon propagator mmc (Muon Monte Carlo) [35], which simulates the muon energy loss during propagation through the ice. The detector response to muons and secondary particles is simulated with the detector simulation amasim [36].

The atmospheric muon simulations reproduce the experimental data well over all analysis levels, up to a normalization factor. Throughout the analysis, the predicted event rate is too low by roughly $10 \%$. This deviation is well within the present uncertainty in the absolute flux of high energy cosmic rays [32], and is compensated by normalizing the simulated background to the number of events found in the $20 \%$ of the experimental data which are not blinded.

\subsection{Simulation of magnetic monopoles}

Magnetic monopole tracks are simulated as an isotropic flux through the detector. The tracks are generated with starting points randomly distributed on a "generation plane" (Fig. 6) perpendicular to the track direction at $1000 \mathrm{~m}$ distance from the detector [29, 37]. Since monopoles with masses in the relevant mass range $\left(M>10^{6} \mathrm{GeV}\right)$ are not substantially slowed down during propagation from the generation point

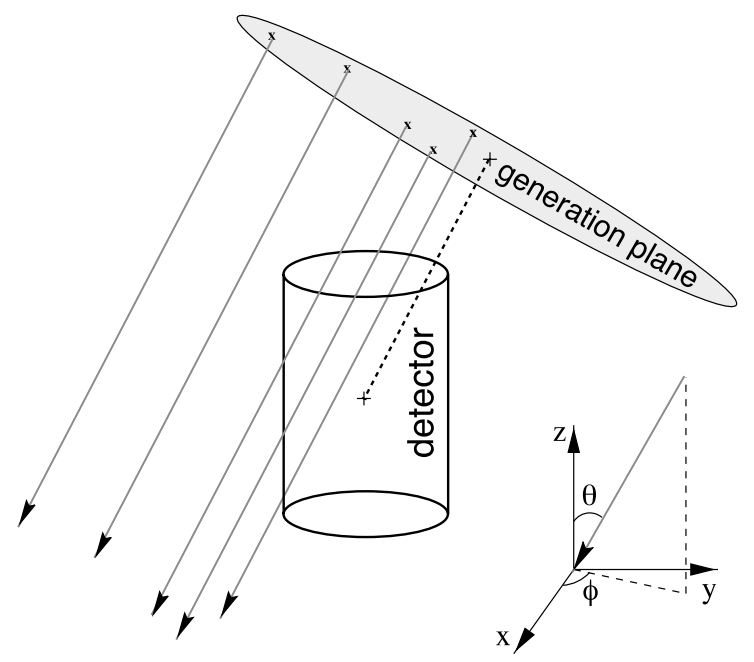

Fig. 6 Simulation of magnetic monopoles. The monopole tracks are generated with starting points randomly distributed about a generation plane which during generation is randomly rotated around the detector center to simulate an isotropic flux to the detector, monopole energy losses within the detection volume are neglected and the monopole speed is assumed to be constant.

Only the direct Cherenkov emissions are simulated. Apart from the direct Cherenkov emission, Cherenkov light from secondary electrically charged particles produced along the monopole track in stochastic energy loss processes (e.g., photo-nuclear interactions or pair production) contributes to the total light yield. The cross sections for these energy loss processes steeply rise with the monopole's Lorentz boost, but the contribution from secondary Cherenkov light is negligible for boosts $\Gamma<10^{4}$ [10]. Neglecting secondary Cherenkov light, the light yield depends only on monopole speed and the monopole charge (equation (2)). The simulation assumes minimally charged magnetic monopoles, carrying one unit Dirac charge. Four different monopole speeds are simulated: $\beta=0.76, \beta=0.8$, $\beta=0.9$, and $\beta=1$. (Although strictly, no massive particle can travel at the vacuum speed of light, the numerical value used for simulating the fastest monopoles is $\beta=1$.)

\section{Data filtering}

Ultimately, experimental and simulated data sets are divided into two subsets containing events with up- and down-going particles respectively. Because the reconstruction of the particle direction is computationally intensive, the data sets are first reduced using fast-to-compute observables that provide a measure for the event light yield. In order to avoid unnecessary loss of signal in the region below the horizon where the background expectation is low, the first selection criteria are designed such that a high fraction of the monopole signal is retained.

\subsection{First level filter}

The highly intense Cherenkov emissions from relativistic magnetic monopoles cause multiple subsequent PMT pulses ("hits") in most of the OMs, while in low energy atmospheric muon events most OMs record only a single hit. The first two selection criteria ("cuts") require (1) at least 140 hits to be recorded in an event and (2) the fraction of fired OMs with only a single hit to be smaller than 0.72 . Figure 7 shows the distributions of the two cut parameters, NHits and FRAC 1, for simulated monopole events, simulated atmospheric muon background, and a small subset of the experimental data set at trigger level. For both cut parameters, the atmospheric muon background decreases more rapidly towards higher (NHits) and lower (FRAC 1) values than the simulated signal. The slopes of the monopole distributions expose a "shoulder", whose position depends on the monopole speed $\beta$. The specific shape of the distributions is 


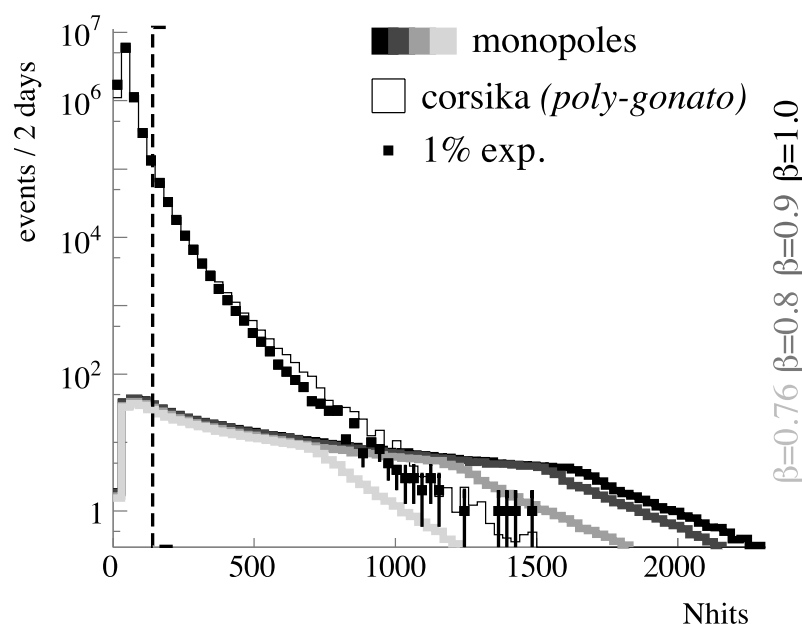

Fig. 7 First level data filter. The cut parameters used are the total number of hits (NHits, left panel) and the fraction of OMs with only a single hit (FRAC 1, right panel). The distributions are shown for trigger level data of simulated atmospheric muons (corsika, black histograms), roughly $1 \%$ of the experimental data set (black markers), and simulated magnetic monopoles with speeds from $\beta=0.76$ (lightest grey histograms) to $\beta \approx 1$ (darkest grey histograms). The experimental data correspond to roughly two days of detector livetime, and simulated

a result of the geometrical shape of the AMANDA-II detector (a tall cylinder), and is common to all observables that provide a measure for the total amount of light deposited in the detector. For monopoles, the Cherenkov emission is constant along the track, and is solely a function of $\beta$ (which is uniform in each of the four simulated monopole event samples). Therefore, for a given $\beta$, the total light deposition of a monopole depends only on the path length it travels through the detector. The monopole events in the tails of the distributions (behind the shoulder) are those monopoles that enter the detector with nearly vertical directions and hence have the longest path length through the instrumented volume.

The cuts are placed such that the experimental data is reduced to an easily manageable size of roughly $1 \%$ of the initially triggered events. The fraction of simulated monopole events that pass the first level filter varies between $75 \%$ $(\beta=0.76)$ and $81 \%(\beta \approx 1)$.

\subsection{Second level filter}

The next selection criterion is an optimized weighted average (Fisher discriminant) of several event observables that are related to the light yield. The Fisher discriminant corresponds to the one-dimensional projection of a multidimensional space of observables, for which signal and background are maximally separated. The method is also known as a linear discriminant analysis (LDA) [38]. The following five event observables are used as input to an LDA:

(1) The number of hit OMs $(\mathrm{NCH})$

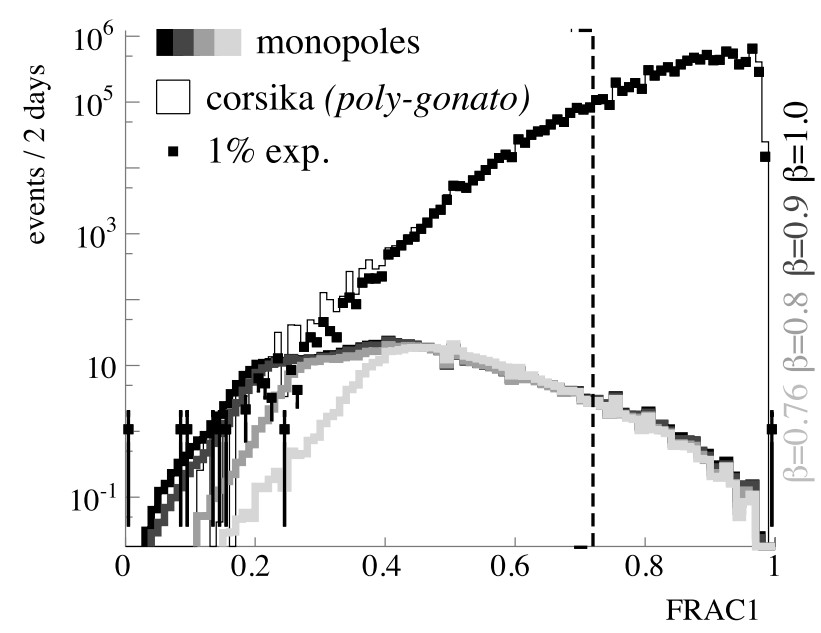

atmospheric muon events are normalized to the number of experimental events. All four magnetic monopole samples are normalized to the same flux of $10^{-13} \mathrm{~cm}^{-2} \mathrm{~s}^{-1} \mathrm{sr}^{-1}$ (two orders of magnitude above the Parker Bound). The four monopole histograms contain different numbers of events, because the Cherenkov light yield increases with $\beta$ (equation (2)), and monopoles with higher speed have a higher probability to fulfill the trigger condition (at least 24 hit OMs). The cuts are marked as dashed black lines

(2) The number of hits (NHits)

(3) The fraction of hit OMs with only a single hit (FRAC 1)

(4) The fraction of hit OMs with optical readout with only a single hit $\left(F R A C 1_{\mathrm{opt}}\right)$.

(5) The "speed of the linefit" $(|\boldsymbol{v}|)$.

The linefit $[39,40]$ is a very fast analytic method to obtain a "first guess" of the particle track parameters. The particle's velocity vector $\boldsymbol{v}$ and the track position $\boldsymbol{r}$ are fit with a $\chi^{2}$ minimization as

$\chi^{2}=\sum_{i}\left(\boldsymbol{r}_{i}-\boldsymbol{r}-\boldsymbol{v} \cdot t_{i}\right)^{2}$,

where the $\boldsymbol{r}_{i}$ and $t_{i}$ are the position and time of $i$ th hit. The linefit does not take into account photon propagation through the ice and is therefore relatively inaccurate, especially for extremely bright events, in which many Cherenkov photons are detected at large distance from the emission point after multiple scatterings. The linefit-speed $|\boldsymbol{v}|$ in particular, cannot be regarded as a measure of the particle speed. It is however related to the light yield, because photons that are delayed by scattering draw the linefit-speed towards smaller values than the particle speed.

Figure 8 shows the Fisher discriminant composed of the five observables (NCH, NHits, FRAC 1, FRAC $1_{\mathrm{opt}}$, and $|\boldsymbol{v}|$ ) that is used as a cut parameter. The cut is placed such that more than $99 \%$ of the remaining monopole signal passes. The looseness of this cut is motivated by the fact that at this filtering level, the particle direction, which is a key criterion to separate the monopole signal from down-going at- 


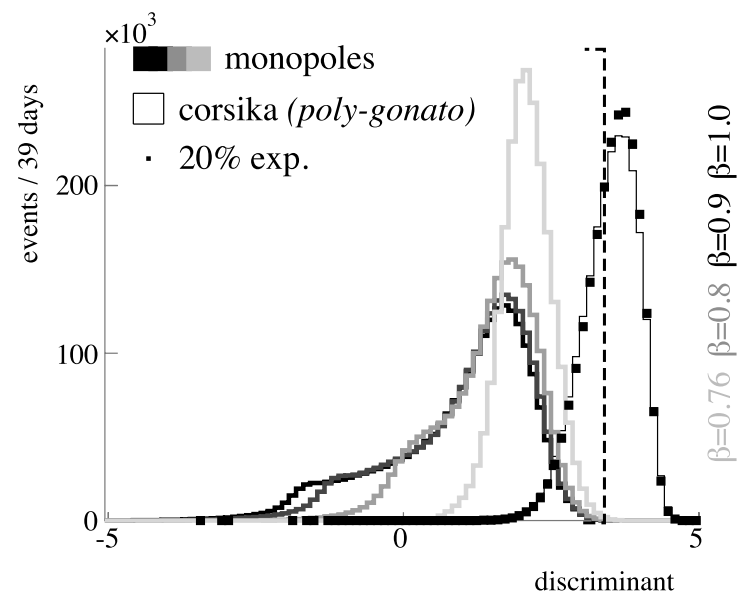

Fig. 8 Fisher discriminant composed of NCH, NHits, FRAC 1, $F R A C 1_{\mathrm{opt}}$, and $|\boldsymbol{v}|$ for $20 \%$ of the experimental data set (corresponding to roughly 39 days of detector livetime, black markers), simulated atmospheric muons (corsika, black histogram), and simulated magnetic monopoles (grey histograms). In order to expose the monopole signal on a linear scale, the monopole samples were normalized to the same number of events as contained in the experimental data set. The dashed black line marks the cut

mospheric muons, has not been reconstructed yet. Therefore, we aim for a minimal loss in signal at this filtering stage. Tighter cuts, which exploit the track directions, are applied at the next filtering level.

\subsection{Track reconstruction and third level filter}

Five track parameters $(\Theta, \Phi, \boldsymbol{r})$ (zenith angle, azimuth, and three spatial coordinates) are fit to the observed set of OM responses $R=\left\{\left(\boldsymbol{r}_{\mathrm{i}}, t_{\mathrm{i}}\right)\right\}$ (spatial coordinates and times of the recorded hits) by minimizing the negative logarithm of the overall likelihood $-\log [\mathcal{L}(R \mid \Theta, \Phi, \boldsymbol{r})]$ [40]. The $\log$ likelihood function is minimized numerically. Starting from a "seed track", the track parameters are varied until a minimum is found. Misreconstruction occurs if the minimization process converges to a local instead to the global minimum. The reconstruction accuracy can be improved by repeating the minimization process multiple times starting from various seed tracks. In case the multiple iterations of the minimization converge to different minima, the track with the lowest value $-\log (\mathcal{L})$ is picked as reconstruction result. However, since the minimization is computationally expensive, the first likelihood reconstruction used in this analysis performs only one minimization process, seeded with the track parameters $(\Theta(v), \Phi(v), r)$ from the linefit. Only after the data volume is further reduced, the minimization is repeated multiple times.

Using a discriminant analysis, the zenith angle of the single-iteration reconstructed track is combined with the FRAC 1 observable (fraction of OMs with only a single hit) to place a cut on the event light yield dependent on the zenith angle of the incoming particles (Fig. 9). Roughly $88 \%$

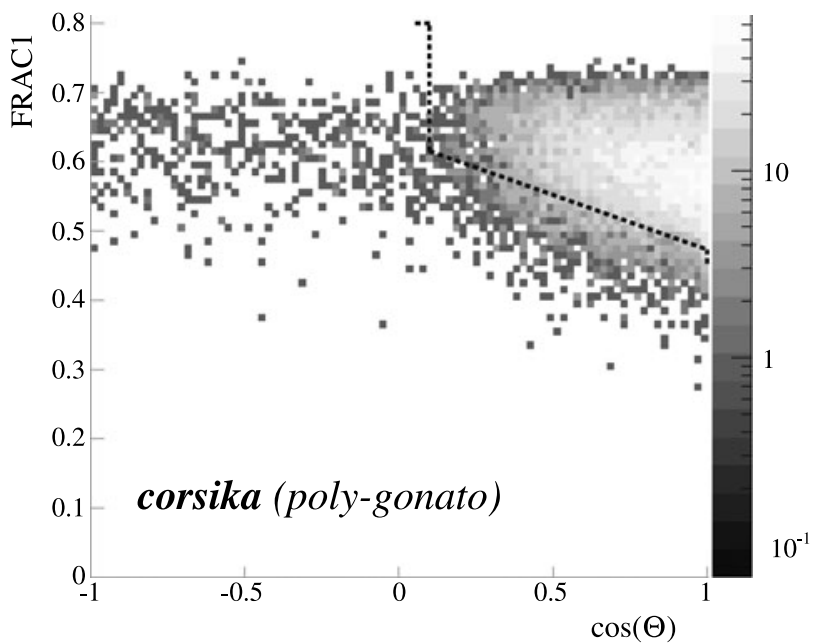

Fig. 9 Correlation between FRAC 1 (fraction of OMs with only a single hit) and the zenith angle $(\cos \Theta$, single-iteration likelihood) for the expected atmospheric muon background (corsika). FRAC 1 is related to the light yield, as brighter events are likely to cause multiple hits in OMs (low value of FRAC 1). The AMANDA-coordinate system is oriented such that $\cos \Theta=1$ corresponds to a vertically downward direction. The dashed line marks the cut in the $\cos \Theta-F R A C 1$-plane

of the remaining atmospheric muon events that are reconstructed with zenith angles of at least $6^{\circ}$ above the horizon $(\cos \Theta>0.1)$ are removed, while $81 \%(\beta=0.76)$ to $91 \%$ $(\beta \approx 1)$ of the signal events in this angular region are preserved.

The minimization of the log-likelihood function is now repeated twelve times using pseudo-random ${ }^{3}$ seeds, and the track with the smallest value of $-\log (\mathcal{L})$ is taken as result. For about $4.9 \%$ of the events remaining in the $20 \%$ of experimental data, this 12-fold reconstruction yields a track zenith angle below the horizon $\left(\Theta>90^{\circ}\right)$, while for simulated background events only $3.4\left( \pm 0.5^{\text {stat }}\right) \%$ are misreconstructed as up-going. This statistically significant underestimation of the misreconstructed atmospheric muon background by the simulation can be ascribed partly to an increase of electronic noise during large signals (predominantly in OMs with electrical readout) which is not reproduced in the simulation and partly to a poor description of the OM response in the upper third of the detector [29]. The poor agreement between experimental and simulated data with respect to the OM response in the upper part of the detector during very bright events is, unfortunately, not understood. In order to enable a blind analysis procedure, the OMs in the upper third of the detector as well as the OMs with electrical readout are discarded, and only optically read out OMs in the lower two thirds of the detector are used for the higher level selection criteria.

\footnotetext{
${ }^{3}$ In order to have reproducible results, the twelve seed tracks are determined deterministically from the result of the preceding minimization using a Sobol sequence (a pseudo-random sequence) [41].
} 
The excess of misreconstructed events in experimental data is removed with an additional light yield criterion that uses only hits in optically read out channels that are located in the lower two thirds of the detector. The cut parameter is the Fisher discriminant composed of $\mathrm{NCH}$ (number hit $\mathrm{OMs}$ ) and NHits (number of hits). Figure 10 shows the reconstructed zenith angle after application of this final cut for simulated signal and background events and for $20 \%$ of the experimental data set.

The following selection criteria are optimized separately for up-going tracks $(\cos \Theta<0)$ and down-going tracks $(\cos \Theta \geq 0)$.

\section{Event selection for up-going tracks}

After applying the above filter criteria, roughly 2000 atmospheric muon events in the blinded data set are expected to be misreconstructed as up-going particles $\left(\Theta>90^{\circ}\right)$.

Atmospheric muon events with extremely high light yield have a higher probability to be misreconstructed than low energy ones: the brightest events either consist of extended bundles of up to several thousands of muons, or, in case the muon multiplicity in the bundle is small, most of the light deposition results from a few large stochastic energy loss processes of individual muons. In both cases, the single track hypothesis that enters the likelihood reconstruction does not provide a good description for the hit pattern, and the log-likelihood function most likely does not possess a pronounced extremum at the true muon direction.

In order to reject misreconstructed high light yield events, we add another iteration of the likelihood reconstruction, using only reduced hit information, namely only hits in the optically read out OMs. The minimization process is seeded with the result of the previous 12 -fold reconstruction. If the seed track fits the observed hit pattern well, the additional iteration should converge to a result similar to the seed. Otherwise the minimizer will probably be "driven away" from the initial track hypothesis.

Requiring that the zenith angle obtained from the last iteration does not deviate by more than $25^{\circ}$ from the one obtained from the 12 -fold reconstruction removes $\sim 40 \%$ of the remaining muon background while $82 \%(\beta \approx 1)$ to $95 \%$ ( $\beta=0.76)$ of the monopole signal is retained.

At the penultimate cut level, we expect roughly $1000 \mathrm{ev}$ ents in the analysis data set. About $35 \%$ of the expected background is induced by primary iron nuclei, roughly $20 \%$ by the light elements (protons and helium nuclei), and $45 \%$ is induced by intermediate-mass nuclei.

The final cut for the monopole search below the horizon is again a light yield criterion. The cut parameter is the sum of the recorded PMT-pulse amplitudes ( $\Sigma A D C$, one peak amplitude for each OM) of all OMs with optical readout that are located in the lower two thirds of the detector

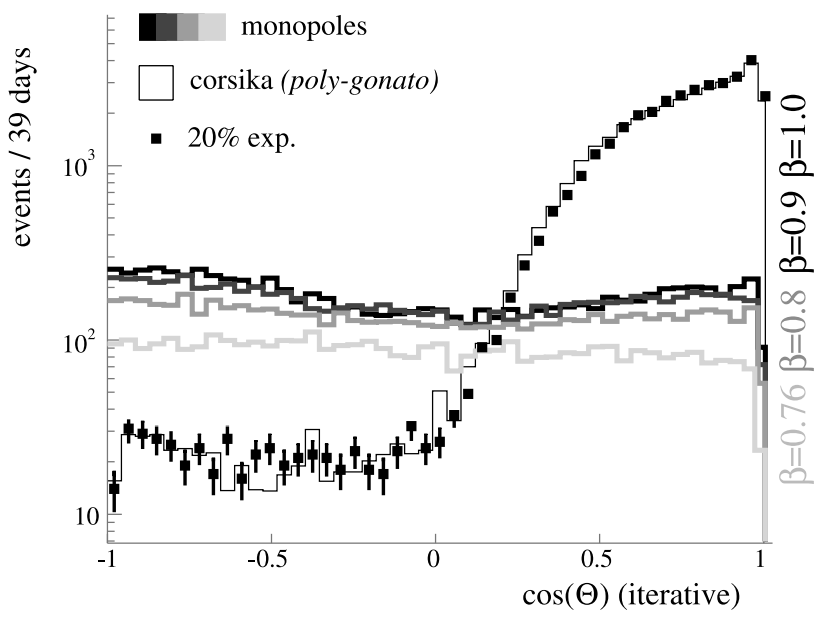

Fig. 10 Cosine of the reconstructed zenith angle obtained from the 12-fold likelihood reconstruction for $20 \%$ experimental data (black markers), simulated atmospheric muon background (black histogram), and simulated magnetic monopoles with an assumed flux of $10^{-13} \mathrm{~cm}^{-2} \mathrm{~s}^{-1} \mathrm{sr}^{-1}$ (two orders of magnitude above the Parker Bound, grey histograms) after application of the third level filter

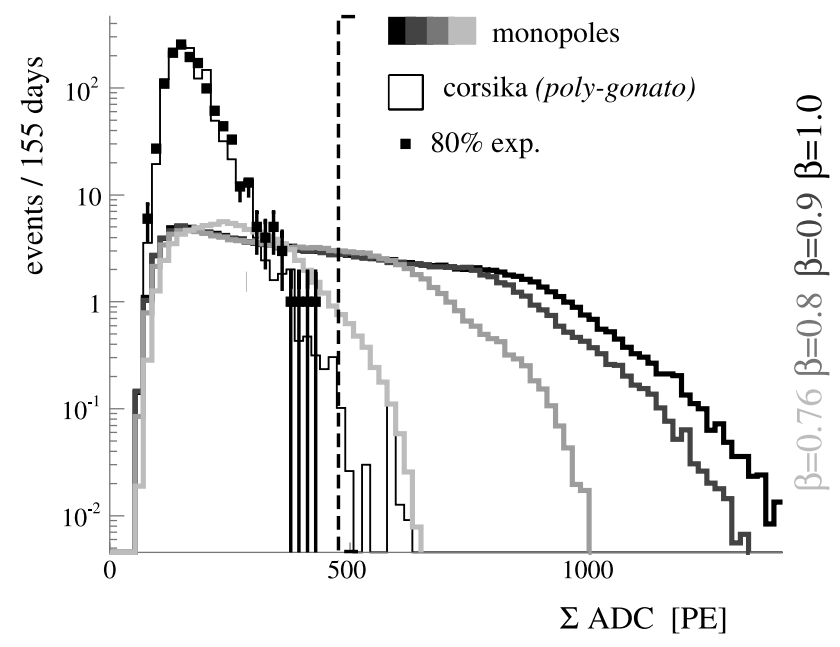

Fig. 11 Distribution of the final cut parameter used for the search for up-going monopoles (the sum of PMT pulse amplitudes measured in OMs with optical readout that are located in the lower two thirds of the detector, $\Sigma A D C$ ) for expected background from atmospheric muons (black histogram), unblinded $80 \%$ of the experimental data (black markers) and simulated monopole signal with an assumed "Parker-flux" of $10^{-15} \mathrm{~cm}^{-2} \mathrm{~s}^{-1} \mathrm{sr}^{-1}$ (grey histograms). The dashed line marks the final cut

(the same set of OMs as used in the last light yield criterion described in the previous section). The cut is optimized to minimize the expected flux limit for $\beta \approx 1$ monopoles at the $90 \%$ confidence level. ${ }^{4}$ The expected background in the blinded $80 \%$ of the data after application of the final cut is 0.23 events. This remaining expected background is mostly

\footnotetext{
${ }^{4}$ This optimization method is referred to as the model rejection potential technique and is described in [42].
} 


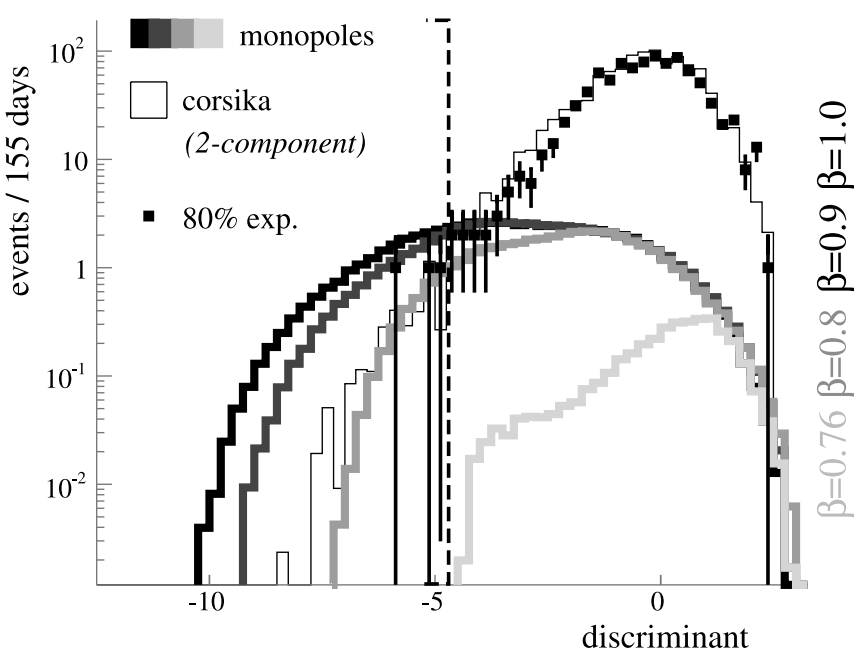

Fig. 12 Final cut used for the monopole search above the horizon. Left: The cut parameter (Fisher discriminant composed of $\cos \Theta$ and $\Sigma A D C$ ) for simulated atmospheric muon background (corsika, black histogram), $80 \%$ experimental data (black markers), and simulated monopoles with an assumed "Parker-flux" of

induced by iron primaries (65\%) and other heavier elements with atomic numbers (nuclear charges) greater than $Z=12$. Of the originally triggered signal events a fraction between $4 \%(\beta=0.76)$ and $11 \%(\beta \approx 1)$ is expected to pass the final cut.

Figure 11 shows the distribution of the final cut parameter $(\Sigma A D C)$ for simulated signal and background events and for the unblinded $80 \%$ of the experimental data set. No experimental event passes the final cut. The non-observation of a monopole candidate allows to place a limit on the flux of magnetic monopoles. The derivation of the resulting limit will be discussed in Sect. 11 together with the result for down-going monopoles.

\section{Event selection for down-going tracks}

The search for magnetic monopoles above the horizon requires more stringent event selection to achieve sufficient reduction of the atmospheric muon background. The used cut parameters are linear combinations (Fisher discriminants) of the zenith angle from the 12-fold likelihood reconstruction and one or more observables that are sensitive to the event light yield. The latter use only optically read out OMs in the lower two thirds of the detector.

Combining the cosine of the reconstructed zenith angle with the number of hits (NHits) and the time span over which the hits are recorded, ${ }^{5}$ reduces the atmospheric muon back-

\footnotetext{
${ }^{5}$ Events with high light yield have many hits from photons that have been delayed by multiple scatterings during propagation over large dis-
}

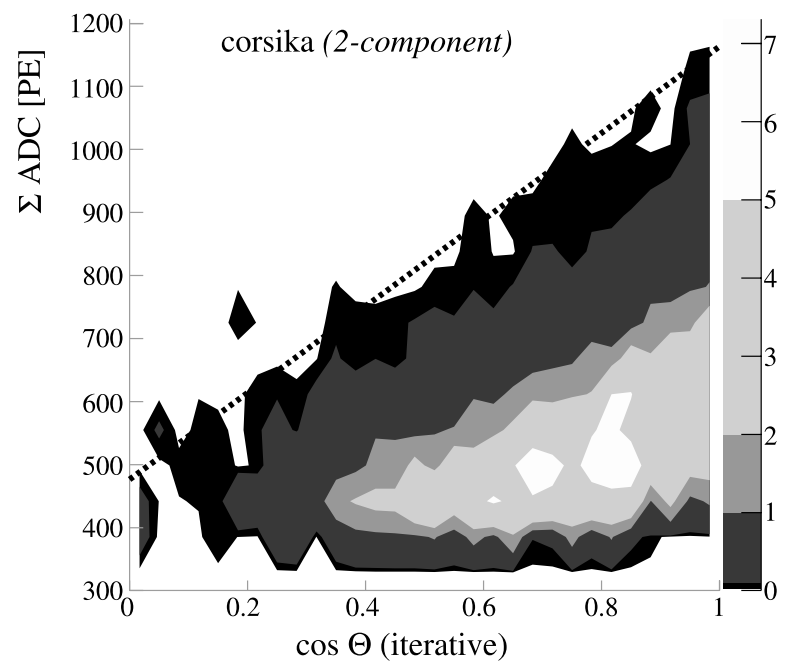

$10^{-15} \mathrm{~cm}^{-2} \mathrm{~s}^{-1} \mathrm{sr}^{-1}$ (grey histograms). The dashed line marks the final cut. Right: The same cut represented as a dashed line in the $\cos \Theta-\Sigma A D C$-plane together with the distribution of the expected atmospheric muon background (corsika)

ground by $97 \%$, while $49 \%(\beta=0.76)$ to $74 \%(\beta \approx 1)$ of the monopole signal is retained.

At the penultimate cut level, we expect roughly $940 \mathrm{ev}-$ ents in the analysis data set. More than $30 \%$ of the remaining background is expected to be induced by cosmic ray nuclei with energies greater than $50 \mathrm{PeV}$, where the corsika simulations using the poly-gonato model do not provide an accurate description of the muon background. Therefore, simulations using the two-component cosmic ray spectrum (see Sect. 6.2) are used to optimize the final cut. According to these simulations, the remaining background at the penultimate cut level is almost solely ( $>95 \%$ ) induced by iron primaries.

The final cut parameter is the Fisher discriminant composed of the cosine of the reconstructed zenith angle with the pulse amplitude sum $(\Sigma A D C)$. The cut is again optimized such that the most stringent limit on $\beta \approx 1$ monopoles is expected. Figure 12 shows the distribution of the final cut parameter for the unblinded experimental data set and for simulated signal and background events. Three events are found after unblinding, consistent with 2.6 events predicted background simulations using the two-component model. ${ }^{6}$

The fraction of simulated signal events that pass the final cut varies between $1 \%(\beta=0.8)$ and $5 \%(\beta \approx 1)$. No simulated monopoles with speed $\beta=0.76$ pass the final cut,

tances. So, the time interval over which light is collected is longer than for events with low light yield.

${ }^{6}$ The corsika simulations using the poly-gonato model on the other hand, predict 0.3 events to pass the final cut, a factor 10 less than the number of events found in the unblinded data. 
Table 1 Relative systematic and statistical error in the predicted event rates at the final analysis level. The systematic error in the signal rate was obtained from Monte Carlo simulations with varied simulation parameters (OM sensitivity, ice properties), while the systematic er- ror in the background rate was estimated using the variations in the "normalization factor" that is applied to the background simulations throughout the analysis (see text)

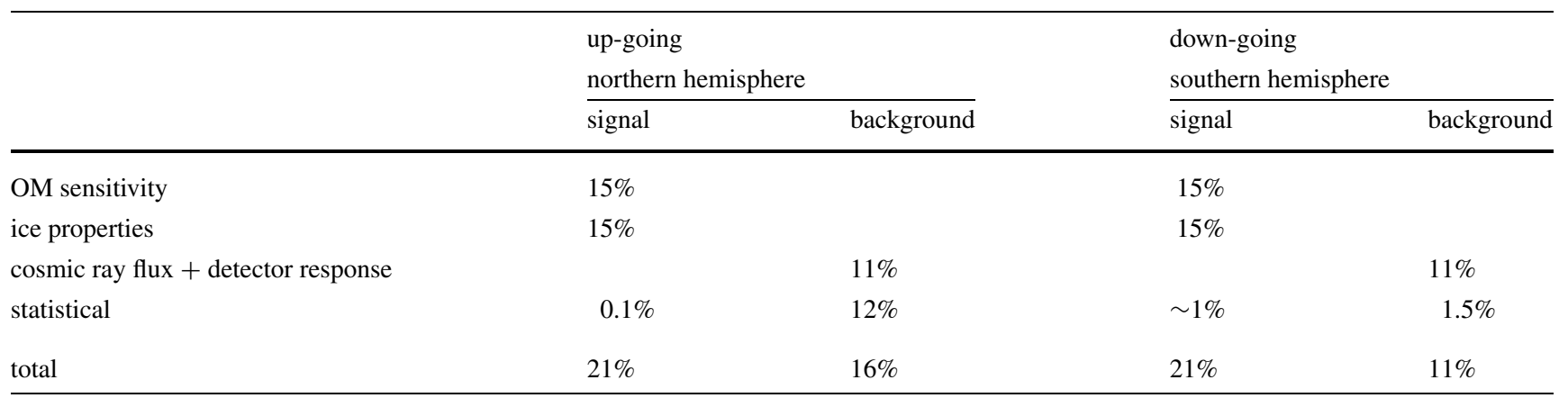

meaning that the search above the horizon has no sensitivity to monopoles with speeds much lower than $\beta=0.8$.

\section{Systematic uncertainties}

For both up- and down-going monopoles, the number of events found in the unblinded experimental data are consistent with the background expectation. The calculation of flux upper bounds takes into account systematic and statistical uncertainties in the background and signal prediction.

Sources of systematic uncertainties in the predicted numbers of signal and background events are mostly imperfections in the detector simulation either due to poor knowledge of the simulation parameters or due to simplifications of physical processes that are necessary to meet limited computing resources. Uncertainties in the absolute OM sensitivity and simplifications of the optical properties of the Polar ice have the largest impact on this analysis.

The deviations of the absolute OM sensitivities from their nominal values were estimated from muon data and are constrained to less than $15 \%$ [43] with the largest deviations observed for OMs on the inner strings with electrical readout, which in this analysis were not used for higher level selection criteria. Studies of variations in the OM sensitivities in the detector simulation showed that the relative change in the predicted event rates is approximately equal to the relative deviation of the OM sensitivities from their nominal value (for a uniform relative deviation for all OMs throughout the detector) or less (for random deviations, see [29] and references therein). We therefore assume that the systematic error in the predicted event rates of this analysis is no bigger than $15 \%$.

Light propagation through the polar ice is simulated adopting depth-averaged optical parameters. For very bright events, the total number of detected photons mostly depends on the mean values of scattering and absorption lengths because variations in the photon collection efficiency average out over the detection volume. The impact of uncertainties of the mean optical parameters in the photon propagation simulation were studied over a wide parameter space [44]. Variations as large as $+12 \% /-30 \%$ (absorption length) and $+8 \% /-21 \%$ (effective scattering length) result in changes of the event rates of $34 \%$ around the average. Since the optical parameters are known to within a few percent [25], we assume the systematic error in the predicted event rates to be no bigger than $15 \%$.

For the total error in the predicted number of signal events, both the systematic error and the statistical error (less than $1 \%$ ) are added in quadrature, resulting in a total error of $21 \%$ (Table 1).

The estimation of the systematic error in the predicted number of background events differs from the systematic error estimation for the signal prediction, because the $20 \%$ of the data which are not blinded provide a reference to adjust the background prediction. By normalizing the number of predicted background events to the number of events observed $20 \%$ of the experimental data, uncertainties in the background rate are partially compensated. Therefore the systematic error in the background rate will be smaller than the systematic error in the signal prediction, for which no such "calibration source" is available.

Intrinsically, the number of predicted background events has additional theoretical uncertainties due to limited knowledge of the absolute flux, the energy spectrum, and to some extent, the composition of high energy primary cosmic rays, as well as uncertainties due to the modeling of high energy hadronic interactions. These unknown parameters would result in a substantial systematic uncertainty in the background rate. ${ }^{7}$ However, throughout the analysis, the

\footnotetext{
${ }^{7}$ The uncertainty in the absolute normalization of the flux for instance is estimated to be about 20\% [32], and uncertainties in the predicted atmospheric muon rate due to the modeling of high energy hadronic interactions are about as large.
} 
Table 2 Cumulative passing rates of simulated monopoles and background events. The rightmost column lists the normalization factor applied to the simulated atmospheric muon background at each cut level, i.e., the ratio of the number of events in the $20 \%$ experimental data and the number of predicted background events

\begin{tabular}{|c|c|c|c|c|c|c|c|}
\hline \multirow[t]{2}{*}{ level } & \multirow[t]{2}{*}{ cut parameter } & \multicolumn{5}{|c|}{ fraction of passing events } & \multirow{2}{*}{$\begin{array}{l}\text { ratio } \\
\text { exp./sim. }\end{array}$} \\
\hline & & $\beta \approx 1$ & $\beta=0.9$ & $\beta=0.8$ & $\beta=0.76$ & corsika & \\
\hline trigger & & & & & & & 1.30 \\
\hline level 1 & NHits, FRAC 1 & 0.81 & 0.81 & 0.80 & 0.75 & 0.01 & 1.22 \\
\hline level 2 & LDA (FRAC 1, FRAC $1_{\mathrm{opt}}$, NHits, NCH, $\left.|\boldsymbol{v}|\right)$ & 0.81 & 0.81 & 0.80 & 0.75 & $5.9 \times 10^{-3}$ & 1.17 \\
\hline level 3 & $\operatorname{LDA}(F R A C 1, \cos \Theta), \operatorname{LDA}(N H i t s, N C H)$ & 0.59 & 0.60 & 0.58 & 0.48 & $1.9 \times 10^{-4}$ & $1.34,1.08$ \\
\hline up-going & $\Theta>90^{\circ}$ & 0.32 & 0.32 & 0.31 & 0.25 & $2.7 \times 10^{-6}$ & 1.12 \\
\hline penultimate & zenith angle difference & 0.26 & 0.26 & 0.27 & 0.24 & $1.6 \times 10^{-6}$ & 1.21 \\
\hline final & $\Sigma A D C$ & 0.11 & 0.10 & 0.08 & 0.04 & $3.2 \times 10^{-10}$ & \\
\hline down-going & $\Theta \leq 90^{\circ}$ & 0.28 & 0.28 & 0.28 & 0.22 & $1.9 \times 10^{-4}$ & 1.08 \\
\hline penultimate & LDA $($ time-span, NHits, $\cos \Theta)$ & 0.20 & 0.20 & 0.19 & 0.11 & $3.8 \times 10^{-6}$ & 1.21 \\
\hline final & $\mathrm{LDA}(\cos \Theta, \Sigma A D C)$ & 0.05 & 0.04 & 0.01 & 0 & $1.1 \times 10^{-8}$ & \\
\hline
\end{tabular}

absolute normalization of the simulated background is determined by scaling the number of predicted background events to the number of events observed in $20 \%$ experimental data. This normalization is adjusted at each selection level, up to the penultimate cut. The normalization after application of the penultimate cut is then kept for optimizing the final cut and for calculating the expected remaining background after unblinding. If systematic errors in the background simulation resulted solely in a scaling of the predicted event rates and did not affect the distributions of the cut parameters qualitatively, the normalization would be constant throughout all selection levels and the systematic uncertainties would be fully compensated. If, on the other hand, systematic errors affect the event observables (i.e., the cut parameters), then the selection efficiencies differ for experimental and simulated data, and the normalization factor (i.e., the ratio between the number of experimental events to the number of predicted background events) varies throughout the selection chain.

Between trigger level and the penultimate selection level of this analysis, the normalization factor varies within $\pm 11 \%$ around an average value of 1.21 (see Table 2 ). We take the $11 \%$ maximum variation in the normalization as a measure for the remaining systematic uncertainty in the background prediction that is not compensated for by scaling (Table 1).

\section{Flux upper limits}

Because of the large range of potential monopole masses and the poor knowledge of the monopole energy loss processes, a generally valid flux limit can only be formulated as a constraint of the flux in the detector. For this analysis,
Table 3 Event upper limits for up- and down-going monopoles. Listed are the expected number of background events in the final data sample $\left\langle n_{\text {bg }}\right\rangle$, the number of observed experimental events $n_{\text {observed }}$, and the resulting event upper limit $\mu_{90 \% \text { C.L., }}$ i.e., the number of signal events that is excluded at the $90 \%$ confidence level. The event upper limits are calculated taking into account statistical and systematic errors [45]

\begin{tabular}{llll}
\hline & $\left\langle n_{\text {bg }}\right\rangle$ & $n_{\text {observed }}$ & $\begin{array}{l}\mu_{90 \% \text { C.L. }} \\
\text { (incl. errors) }\end{array}$ \\
\hline up-going & $0.23 \pm 0.04$ & 0 & 2.4 \\
down-going & $2.61 \pm 0.29$ & 3 & 5.2 \\
\hline
\end{tabular}

this constraint is a limit on an isotropic flux of monopoles with a given speed at the AMANDA-II depth.

The flux limit calculation takes into account the uncertainties in signal and background predictions following the method described in [45]. Table 3 lists the event upper limits at the $90 \%$ confidence level ( $\left.\mu_{90 \% \text { C.L. }}\right)$ for both up- and down-going monopole searches.

The corresponding flux limits for each monopole speed $\beta$ are calculated from the event upper limits as

$\Phi_{90 \% \mathrm{C} . \mathrm{L}}^{\beta}=\frac{\mu_{90 \% \mathrm{C} . \mathrm{L} .}}{\left\langle n_{\mathrm{sig}}^{\beta}\right\rangle} \cdot \Phi_{\text {simulated }}^{\beta}$,

where $\left\langle n_{\text {sig }}^{\beta}\right\rangle$ is the number of expected signal events for a given simulated flux $\Phi_{\text {simulated }}^{\beta}$. The flux limits obtained from the searches in the northern and southern hemispheres are listed in Table 4. These limits apply to fluxes of monopoles with the respective speed in the AMANDA-II detector.

Figure 13 shows the flux limits in comparison to the best presently available experimental bounds. In the velocity range $\beta>0.8$, the limits for monopoles entering from below 
Table 4 Limits on the flux of magnetic monopoles with speeds between $\beta=0.76$ and $\beta \approx 1$ obtained from searches in the northern and southern hemispheres, respectively. Systematic uncertainties are accounted for according to [45]

\begin{tabular}{|c|c|c|}
\hline \multirow[t]{2}{*}{ speed $\boldsymbol{\beta}$} & \multicolumn{2}{|c|}{$\Phi_{90 \% \text { C.L }}\left[\mathrm{cm}^{-2} \mathrm{~s}^{-1} \mathrm{sr}^{-1}\right]$} \\
\hline & $\begin{array}{l}\text { up-going } \\
\text { (northern hemisphere) }\end{array}$ & $\begin{array}{l}\text { down-going } \\
\text { (southern hemisphere) }\end{array}$ \\
\hline 0.76 & $8.8 \times 10^{-16}$ & \\
\hline 0.8 & $6.7 \times 10^{-17}$ & $1.7 \times 10^{-15}$ \\
\hline 0.9 & $4.3 \times 10^{-17}$ & $4.2 \times 10^{-16}$ \\
\hline 1.0 & $3.8 \times 10^{-17}$ & $2.9 \times 10^{-16}$ \\
\hline
\end{tabular}

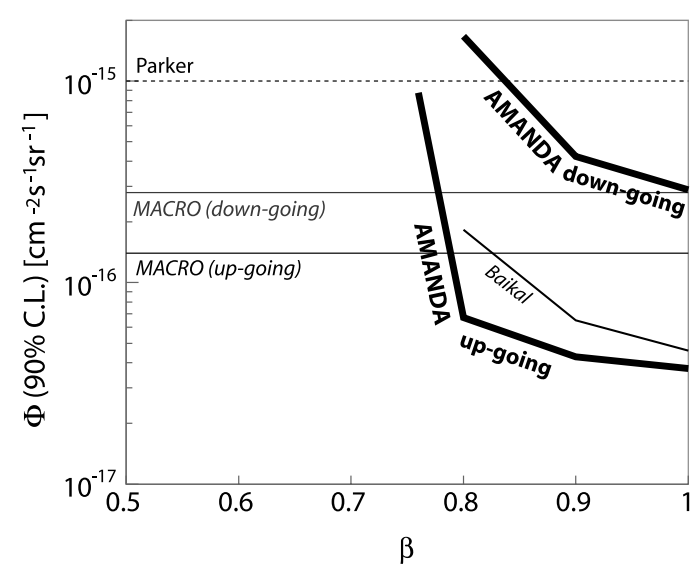

Fig. 13 Limits on the flux of minimally charged relativistic magnetic monopoles with speeds $0.5<\beta<1$. The thick black lines are the limits for up- and down-going monopoles obtained from this work. The thin black lines are the limits on the flux of up-going monopoles published by the Baikal neutrino telescope [15] and the MACRO experiment [14]. The grey horizontal line is the MACRO limit for $2 \pi \mathrm{sr}$ acceptance (down-going monopoles). The dashed line is the Parker Bound [12]

the horizon (northern hemisphere) are the most stringent experimental limits at the time of this writing. The monopole search above the horizon (southern hemisphere) yielded flux limits for $\beta \approx 1$ monopoles at roughly the same level as comparable searches undertaken by the Monopole, Astrophysics and Cosmic Ray Observatory (MACRO) [14]. The search above the horizon is however not sensitive to monopoles with speeds smaller than $\beta=0.8$.

\section{Effective area}

Because very light and ultra-relativistic monopoles can only be detected in a limited angular region (above or around the horizon, see Fig. 3), it is important to study the monopole detection efficiency as a function of the zenith angle of the incoming monopoles. A practical measure of the detection efficiency is the detector effective area, $A_{\text {eff }}$, which is calcu-

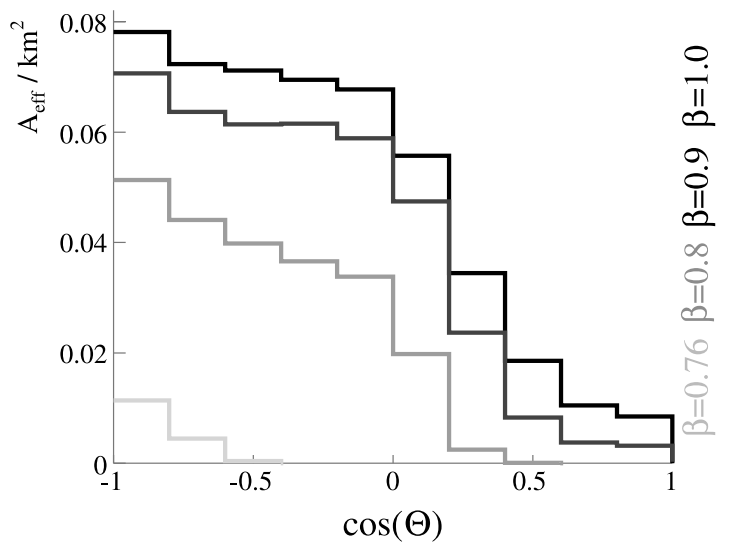

Fig. 14 Detector effective area for relativistic magnetic monopoles arriving at the detector with speed $\beta=0.76$ (lightest grey) to $\beta \approx 1$ (darkest grey) as a function of the zenith angle. ( $\cos \Theta=-1$ corresponds to a vertically up-going monopole in AMANDA-II)

lated as

$A_{\text {eff }}(\beta, \Theta)=\frac{N_{\text {detected }}(\beta, \Theta)}{N_{\text {generated }}(\beta, \Theta)} \cdot A_{\text {gen }}$,

where $N_{\text {detected }}(\Theta, \beta)$ and $N_{\text {generated }}(\Theta, \beta)$ are the numbers of detected and generated monopoles with speed $\beta$ and zenith angle $\Theta$, respectively, and $A_{\text {gen }}$ is the area of the generation plane. The effective area corresponds to the cross sectional area of an ideal detector with $100 \%$ efficiency. Figure 14 shows the effective area as a function of the zenith angle $(\cos \Theta)$ at the final analysis level.

The effective area is highest for vertically up-going monopoles $(\cos \Theta=-1)$ and falls continuously with the zenith angle. This is a result of both the geometry of the AMANDAII detector and the zenith angle dependent event selection. Below the horizon, cuts are based solely on the amount of light deposited in the detector, which in turn depends on the particle's path length through the detection volume. For a tall cylindrical detector, the path is longest in the vertical direction. Therefore, the efficiency is highest in the vertical direction, although the cross-sectional area of the detector is smallest. Above the horizon, on the other hand, the cuts on the light deposition are tightened as a function of the zenith angle, resulting in a decrease of the detection efficiency towards vertical directions.

The effective area shown in Fig. 14 applies to monopoles that are detected via their direct Cherenkov emissions only. For ultra-relativistic monopoles, the direct emissions are substantially enhanced by Cherenkov light from relativistic electrically charged secondary particles produced in stochastic energy loss processes. Since the data selection in this analysis is mostly based on the event light yield, we can reasonably assume that the detection efficiency for ultrarelativistic monopoles is in effect higher. 


\section{Universal flux limit and validity range}

A translation of the limits on the monopole flux through the AMANDA-II detector into a limit on the monopole flux in the vicinity of the Earth is not straightforward. The speed of a monopole reaching the detector depends on its initial kinetic energy, its angle of incidence, and on the monopole's energy loss during its passage through the Earth, which in turn depends on the monopole's Lorentz boost and hence its mass. The monopole mass is essentially an open parameter, as predictions from various unified theories span many orders of magnitudes. For the monopole energy loss, only the electromagnetic part is understood well enough to be treated quantitatively [10]. The least known parameter is the kinetic energy distribution of monopoles.

In order to interpret our limits on the local flux in AMANDA-II as limits on an isotropic monopole flux in the vicinity of the Earth, we calculate approximate limits on hypothetical fluxes of mono-energetic, minimally charged monopoles with masses $10^{5} \mathrm{GeV}<M<10^{18} \mathrm{GeV}$ and kinetic energies of $10^{5} \mathrm{GeV}<E_{\text {kin }}<10^{18} \mathrm{GeV}$.

For a hypothetical isotropic flux $\Phi^{M, E_{\mathrm{kin}}}$ of monopoles with constant initial kinetic energy (and constant initial Lorentz boost $\Gamma_{\text {initial }}$ ), the number of signal events that are expected to be detected within the zenith angle range $\left[\cos \Theta_{\min }, \cos \Theta_{\max }\right]$ is

$$
\begin{aligned}
\left\langle n_{\mathrm{sig}}\right\rangle= & \Phi^{M, E_{\mathrm{kin}}} \cdot T_{\text {live }} \cdot 2 \pi \int_{\cos \Theta_{\mathrm{min}}}^{\cos \Theta_{\mathrm{max}}} d \cos \Theta \\
& \times \int_{0}^{\infty} d \Gamma_{\text {final }} P^{\Gamma_{\text {initial }} \rightarrow \Gamma_{\text {final }}}(\cos \Theta) \\
& \times A_{\mathrm{eff}}\left(\Gamma_{\text {final }}, \cos \Theta\right)
\end{aligned}
$$

where $T_{\text {live }}$ is the effective detector livetime, $P^{\Gamma_{\text {initial }} \rightarrow \Gamma_{\text {final }}}(\cos \Theta)$ is the probability that a monopole with initial Lorentz boost $\Gamma_{\text {initial }}$ and zenith angle $\Theta$ arrives at the detector with a boost factor $\Gamma_{\text {final }}$, and $A_{\text {eff }}$ is the detector effective area (equation (6)).

The function $A_{\mathrm{eff}}\left(\Gamma_{\mathrm{final}}, \cos \Theta\right)$ is zero for $\Gamma_{\text {final }}<1.51$ (corresponding to speeds below the Cherenkov threshold $\beta=0.75)$. For higher $\Gamma_{\text {final }}$, we conservatively approximate the effective area as a step function as $A_{\text {eff }}(\beta=0.76)$ for $1.51<\Gamma<1.67, A_{\mathrm{eff}}(\beta=0.8)$ for $1.67 \leq \Gamma<2.29$, $A_{\text {eff }}(\beta=0.9)$ for $2.29 \leq \Gamma<10$, and $A_{\text {eff }}(\beta \approx 1)$ for $\Gamma \geq 10$.

If we further neglect the stochastic nature of the energy loss, and approximate the total energy loss of a magnetic monopole by the average total electromagnetic energy loss during its passage through the Earth, we can write

$$
\begin{aligned}
& P^{\Gamma_{\text {initial }} \rightarrow \Gamma_{\text {final }}(\cos \Theta)} \\
& \quad=\delta\left(\Gamma_{\text {initial }}-\Gamma_{\text {final }}-\frac{\Delta E_{\text {total }}^{\text {e.m. }}\left(\cos \Theta, \Gamma_{\text {initial }}\right)}{M}\right) .
\end{aligned}
$$

The total energy loss $\Delta E_{\text {total }}^{\text {e.m. }}\left(\cos \Theta, \Gamma_{\text {initial }}\right)$ is again obtained by integrating the approximate formulas for the electromagnetic energy loss [10] over the Earth's matter profile (see Fig. 2).

The limit on a flux of monopoles with a given mass and kinetic energy, $\Phi_{90 \% \text { C.L. }}^{M, E_{\mathrm{kin}}}$ is then

$\Phi_{90 \% \text { C.L. }}^{M, E_{\text {kin }}}=\frac{\mu_{90 \% \text { C.L }}}{\left\langle n_{\text {sig }}\right\rangle} \cdot \Phi^{M, E_{\text {kin }}}$,

where $\mu_{90 \% \text { C.L }}$ is the event upper limit, and $\left\langle n_{\text {sig }}\right\rangle$ is calculated using (7) and (8). For the search for up-going monopoles (search in the northern hemisphere) the zenith angle integral in (7) runs from $\cos \Theta_{\min }=-1$ to $\cos \Theta_{\max }=0$ and the event upper limit is $\mu_{90 \% \text { C.L }}=2.4$ (see Table 3). For the search for down-going monopoles (search in the southern hemisphere) we integrate from $\cos \Theta_{\min }=0$ to $\cos \Theta_{\max }=1$ and the event upper limit is $\mu_{90 \% \text { C.L }}=5.2$.

Of both analyses, only the search for up-going monopoles yields competitive limits. Figure 15 compares the flux

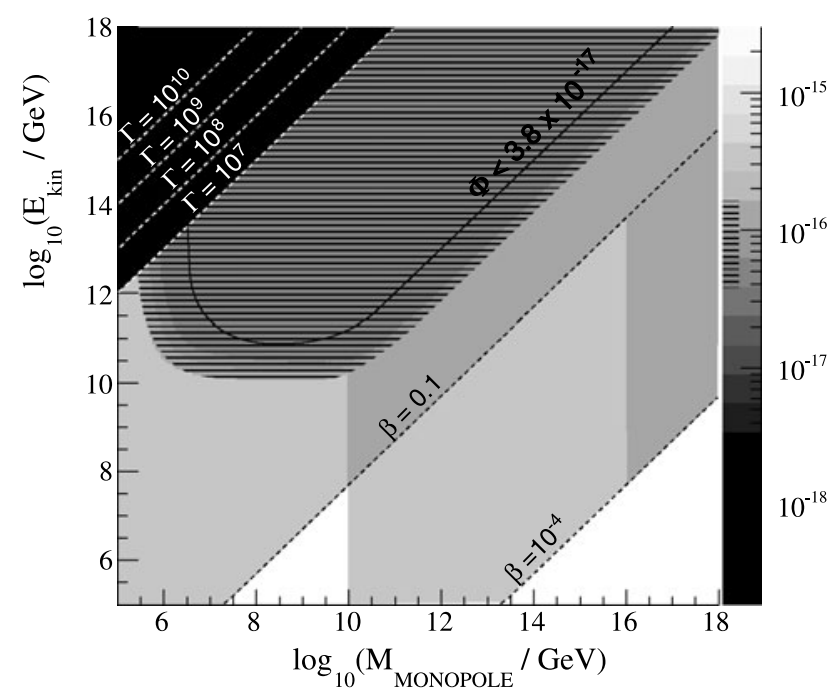

Fig. 15 Limits on hypothetical isotropic and mono-energetic monopole fluxes in the vicinity of the Earth for various monopole masses (horizontal axis) and initial kinetic energies (vertical axis). The grey scale of the colored areas represents the flux level in units of $\mathrm{cm}^{-2} \mathrm{~s}^{-1} \mathrm{sr}^{-1}$ that are constraint at the $90 \%$ confidence level. The hatched area is the region in which the AMANDA-II limit (this work) is the most stringent limit to date. The black contour within this region defines the parameter space over which monopoles reach the AMANDA-II detector with a Lorentz boost $\Gamma>10$. Within this region the flux limit of $3.8 \times 10^{-17} \mathrm{~cm}^{-2} \mathrm{~s}^{-1} \mathrm{sr}^{-1}$ (obtained for $\beta \approx 1$ monopoles) applies. The flux of very light and ultra-relativistic monopoles ( $\Gamma>10^{7}$, black region) is constrained to a level of at least one order of magnitude below the AMANDA-II limit by the RICE experiment [46]. The light grey areas extending to lower kinetic energies and higher monopole masses are the regions over which the monopole flux is constrained to a level of $2.8 \times 10^{-16} \mathrm{~cm}^{-2} \mathrm{~s}^{-1} \mathrm{sr}^{-1}\left(\beta>0.1\right.$ and $\beta>10^{-4}, M>10^{10} \mathrm{GeV}$, lighter grey) and $1.4 \times 10^{-16} \mathrm{~cm}^{-2} \mathrm{~s}^{-1} \mathrm{sr}^{-1}\left(\beta>0.1, M>10^{10} \mathrm{GeV}\right.$ and $\beta>10^{-4}, M>10^{16} \mathrm{GeV}$, darker grey) by the MACRO experiment [14] 
limits obtained from this work (up-going monopoles) to the currently most stringent limits from other experiments. The hatched area in the figure represents the region of potential monopole masses and kinetic energies for which the AMANDA-II limits are the most stringent limits as of today. This region covers relativistic monopoles with initial kinetic energies above $\sim 10^{10} \mathrm{GeV}$ and initial Lorentz boosts smaller than $\Gamma_{\text {initial }} \sim 10^{7}$. (Note that this region may be smaller if a substatial fraction of the monopole energy loss is due to hadronic interactions, which we have neglected in our calculations.) For lower initial kinetic energies, the AMANDA-II limits are outpaced by the limits placed by (MACRO) [14]. The MACRO detector relied on ionization of liquid scintillator or streamer tube gas and was therefore sensitive to monopoles with lower speeds and larger masses. For Lorentz boosts $\Gamma_{\text {initial }}>10^{7}$, our limits are outpaced by the limits set by the Radio Ice Cherenkov Experiment (RICE) [46]. The RICE limits lie between $1.9 \times 10^{-19}$ and $2.2 \times 10^{-18} \mathrm{~cm}^{-2} \mathrm{~s}^{-1} \mathrm{sr}^{-1}$ (depending on $\Gamma$ ), more than one order of magnitude below our limits.

\section{Summary and outlook}

Data taken with the AMANDA-II neutrino telescope during the year 2000 were searched for the Cherenkov signature of relativistic magnetic monopoles. Data selection criteria were developed based on simulated atmospheric muon events (the dominant background to this search) and simulated monopole events, in which the monopoles were assumed to carry one unit of Dirac charge and to travel at fractions of the vacuum speed of light between $\beta=v / c=0.76$ and $\beta \approx 1$. No monopole signal was found in approximately 155 days of detector live time, and upper limits on the monopole flux were derived.

Two separate flux limits were derived for monopoles entering from below and from above the horizon (southern and northern hemisphere), respectively. The analysis considering monopoles entering from below the horizon constrains the flux of magnetic monopoles through the detector to levels between $3.8 \times 10^{-17} \mathrm{~cm}^{-2} \mathrm{~s}^{-1} \mathrm{sr}^{-1}(\beta \approx 1)$ and $8.8 \times 10^{-16} \mathrm{~cm}^{-2} \mathrm{~s}^{-1} \mathrm{sr}^{-1}(\beta=0.76)$ at the $90 \%$ confidence level. Because of the much larger background from downgoing atmospheric muons, the analysis considering monopoles entering from above the horizon yielded limits less stringent by roughly an order of magnitude.

The analysis of only one year of AMANDA-II data allowed to improve current experimental bounds on the flux of relativistic magnetic monopoles. Although several more years of AMANDA-II data are available, future monopole searches will focus on data taken with the IceCube detector [47-49], a cubic-kilometer-scale neutrino telescope that is presently being constructed at the South Pole. First monopole simulations of an intermediate construction stage of the detector, in which almost a third of the optical modules are deployed ${ }^{8}$ indicate that the larger detection volume and improved hardware will enable more advanced signal selection techniques using not only event observables related to the Cherenkov light yield but also to the monopole speed. The sensitivity of this future analysis is expected to be more than one order of magnitude below the flux limits presented here [50].

Acknowledgements We acknowledge the support from the following agencies: US National Science Foundation-Office of Polar Programs, US National Science Foundation-Physics Division, University of Wisconsin Alumni Research Foundation, US Department of Energy, and National Energy Research Scientific Computing Center, the Louisiana Optical Network Initiative (LONI) grid computing resources; National Science and Engineering Research Council of Canada; Swedish Research Council, Swedish Polar Research Secretariat, Swedish National Infrastructure for Computing (SNIC), and Knut and Alice Wallenberg Foundation, Sweden; German Ministry for Education and Research (BMBF), Deutsche Forschungsgemeinschaft (DFG) SFB-676, Research Department of Plasmas with Complex Interactions (Bochum), Germany; Fund for Scientific Research (FNRSFWO), FWO Odysseus programme, Flanders Institute to encourage scientific and technological research in industry (IWT), Belgian Federal Science Policy Office (Belspo); Marsden Fund, New Zealand; Japan Society for Promotion of Science (JSPS); the Swiss National Science Foundation (SNSF), Switzerland; A. Groß acknowledges support by the EU Marie Curie OIF Program; J.P. Rodrigues acknowledges support by the Capes Foundation, Ministry of Education of Brazil.

Open Access This article is distributed under the terms of the Creative Commons Attribution Noncommercial License which permits any noncommercial use, distribution, and reproduction in any medium, provided the original author(s) and source are credited.

\section{References}

1. P.A.M. Dirac, Proc. R. Soc. A 133, 60-72 (1931)

2. H. Georgi, S.L. Glashow, Phys. Rev. Lett. 32, 438-441 (1974)

3. G. 't Hooft, Nucl. Phys. B 79, 276-284 (1974)

4. A.M. Polyakov, JETP Lett. 20, 194-195 (1974)

5. T.W.B. Kibble, J. Phys. A 9, 1387-1398 (1976)

6. H. Georgi, H.R. Quinn, S. Weinberg, Phys. Rev. Lett. 33, 451-454 (1974)

7. M. Daniel, G. Lazarides, Q. Shafi, Nucl. Phys. B 170, 156 (1980)

8. G. Lazarides, C. Panagiotakopoulos, Q. Shafi, Phys. Rev. Lett. 58, 1707 (1987)

9. T.W. Kephart, Q. Shafi, Phys. Lett. B 520, 313-316 (2001)

10. S.D. Wick, T.W. Kephart, T.J. Weiler, P.L. Biermann, Astropart. Phys. 18, 663-687 (2003)

11. J. Preskill, Ann. Rev. Nucl. Part. Sci. 34, 461-530 (1984)

12. M.S. Turner, E.N. Parker, T.J. Bogdan, Phys. Rev. D 26, 1296 (1982)

13. D.E. Groom, Phys. Rep. 140, 323 (1986)

14. M. Ambrosio et al., Eur. Phys. J. C 25, 511 (2002)

15. K. Antipin et al., Astropart. Phys. 29, 366 (2008)

16. S.P. Ahlen, Rev. Mod. Phys. 52, 121 (1980)

\footnotetext{
${ }^{8}$ This intermediate detector configuration is referred to as IceCube-22. It corresponds to the construction stage during the year 2007, in which 22 of the 80 foreseen strings were deployed.
} 
17. D.R. Tompkins, Phys. Rev. B 138, 248-250 (1965)

18. V.A. Rubakov, Rep. Prog. Phys. 51, 189-241 (1988)

19. C.G. Callan, Phys. Rev. D 25, 2141 (1982)

20. A. Pohl, Uppsala University, Ph.D. thesis, 2009

21. A. Pohl, D. Hardtke, in Proc. 30th ICRC, Merida, Mexico (2007)

22. http://pdg.lbl.gov/2009/AtomicNuclearProperties

23. E. Andres et al., Nature 410, 441-443 (2001)

24. E.W. Kolb, M.S. Turner, The Early Universe (Addison Wesley, Reading, 1990)

25. M. Ackermann et al., J. Geophys. Res. 111D, 13203 (2006)

26. E. Andres et al., Astropart. Phys. 13, 1 (2000)

27. A. Karle, Nucl. Instrum. Methods A 387, 274-277 (1997)

28. W.-M. Yao et al., J. Phys. G 33, 1-1232 (2006)

29. H. Wissing, RWTH Aachen University, Ph.D. thesis (2009)

30. D. Heck et al., Forschungszentrum Karlsruhe, Tech. Rep. FZK 6019 (1998)

31. N.N. Kalmykov, S.S. Ostapchenko, A.I. Pavlov, Nucl. Phys. Proc. Suppl. 52B, 17-28 (1997)

32. J.R. Hoerandel, Astropart. Phys. 19, 193-220 (2003)

33. R. Glasstetter et al., in Proc. 26th ICRC, Salt Lake City, USA (1999)

34. T. Antoni et al., Nucl. Instrum. Methods A 513, 490-510 (2003)

35. D. Chirkin, W. Rhode, in Proc. 27th ICRC, Hamburg, Germany (2001)
36. S. Hundertmark, Humboldt-Universität zu Berlin, Ph.D. thesis (1999)

37. C.H.V. Wiebusch, Technical report (1998). http://www.physto. se/ amanda/local/siegmund/gen_2.html

38. R.A. Fisher, Ann. Eugen. 7, 179-188 (1936)

39. V.J. Stenger, University of Hawaii at Manoa, External Report, HDC-1-90 (1990)

40. J. Ahrens et al., Nucl. Instrum. Methods A 524, 169-194 (2004)

41. W.H. Press et al., Numerical Recipes (Cambridge University Press, Cambridge, 1992)

42. G.C. Hill, K. Rawlins, Astropart. Phys. 19, 393-402 (2003)

43. R. Lang, Diploma thesis, Faculty of Sciences, University of Ulm (2005)

44. M. Ackermann et al., Astropart. Phys. 22, 339-353 (2005)

45. J. Conrad et al., Phys. Rev. D 67, 012002 (2003)

46. D.P. Hogan, D.Z. Besson, J.P. Ralston, I. Kravchenko, D. Seckel, Phys. Rev. D 78, 075031 (2008)

47. J. Ahrens et al., Astropart. Phys. 20, 507-532 (2004)

48. J. Ahrens et al., IceCube Preliminary Design Document (2004). http://www.icecube.wisc.edu/science/publications/pdd

49. R. Abbasi et al., Nucl. Instrum. Methods A 601, 294 (2009)

50. B. Christy, University of Maryland at College Park, Ph.D. thesis (in preparation) 